\title{
Experimentelle Untersuchung von Einflussgrößen auf das Rasselgeräuschverhalten von Stirnrädern
}

\author{
Christian Namhoff' ${ }^{1}$ (D) Marcel Kasten $^{1}$ (D) $\cdot$ Jens Brimmers ${ }^{1}$ (D) $\cdot$ Christian Brecher $^{1}$
}

Eingegangen: 8. Oktober 2020 / Angenommen: 5. Februar 2021 / Online publiziert: 3. März 2021

(c) Der/die Autor(en) 2021

\section{Zusammenfassung}

Die Reduzierung von Störgeräuschen spielt eine wesentliche Rolle im Entwicklungsprozess eines Automobils. Neben dem tonalen Getriebeheulen ist das impulsartige Rasselgeräusch in der Fahrerkabine relevant. Das Getrieberasseln entsteht durch eine Drehungleichförmigkeit im Triebstrang, die durch den ungleichförmigen Verbrennungsprozess des Verbrennungsmotors aufgeprägt wird. Die Zahnflanken heben bei Überschreitung einer bestimmten Winkelbeschleunigungsamplitude voneinander $a b$ und bewegen sich innerhalb ihres Verdrehflankenspiels, sodass Schwingungsstöße entstehen. In diesem Bericht wird die experimentelle Untersuchung der Einflüsse Anregungsfrequenz, Schleppmoment und Schmierstoff auf das Rasselgeräuschverhalten von Stirnrädern vorgestellt. Die Rasselgrenze ist durch einen signifikanten Anstieg im Körperschall- und Drehbeschleunigungspegel sowie durch Impulse im Zeitverlauf des Körperschalls und der Drehbeschleunigung zu erkennen. Die Anregungsfrequenz der Drehzahlschwankung beeinflusst die Anzahl an Verdrehflankenstößen pro Umdrehung und führt tendenziell bei einer Zunahme zu einer verringerten Rasselgrenze. Nach Überschreiten der Rasselgrenze erfolgt eine Annäherung der Körperschallpegel von aufgeprägten Drehzahlschwankungen unterschiedlicher Anregungsfrequenzen und ab einer bestimmten Drehzahlschwankungsamplitude sind die Körperschallpegel miteinander vergleichbar. Bei steigendem Schleppmoment nimmt die Drehzahlschwankungsamplitude tendenziell zu, bei welcher die Prüfverzahnung anfängt zu rasseln. Die Rasselgrenze wird durch das Schleppmoment beeinflusst - nach dem Überschreiten der Rasselgrenze weichen jedoch weder die Körperschallpegel noch die Drehbeschleunigungspegel bei unterschiedlich wirkenden Schleppmomenten signifikant voneinander ab. Der Schmierstoff zeigt bei den gewählten Versuchsparametern den höchsten Einfluss auf das Rasselgeräuschverhalten der Prüfverzahnung beim Vergleich der Schmierstoffe SAE 0W-16 und SAE 85W-90. Die Körperschallverläufe weisen im Zeitbereich bei Betrachtung der Abklingkurven bei konstanter Drehzahlschwankungsamplitude auf eine höhere Dämpfung des Schmierstoffs SAE 85W-90 mit höherer Schmierstoffviskosität hin. Der Dämpfungseffekt des Öls nimmt mit zunehmender Anregungsfrequenz bei konstanter Drehzahlschwankungsamplitude ab.

Christian Namhoff

c.namhoff@wzl.rwth-aachen.de

Marcel Kasten

m.kasten@wzl.rwth-aachen.de

Jens Brimmers

j.brimmers@wzl.rwth-aachen.de
Christian Brecher

c.brecher@wzl.rwth-aachen.de

1 Lehrstuhl für Werkzeugmaschinen, Werkzeugmaschinenlabor WZL der RWTH Aachen University, Campus-Boulevard 30, 52074 Aachen, Deutschland 


\title{
Experimental investigation of the influencing factors on the gear rattle noise behavior of cylindrical gears
}

\begin{abstract}
The reduction of annoying sounds is crucial within the vehicle development process. Apart from gear whining, gear rattling is relevant within the driver's cabin. Gear rattling occurs due to a speed non-uniformity within the drive train, caused by the irregular combustion process of the combustion engine. The tooth flanks lose contact and move within the gear backlash when exceeding a certain angular acceleration amplitude, leading to vibro-impacts. This report examines the effects of the excitation frequency of the speed non-uniformity, the drag torque and the lubricant on the gear rattle noise of cylindrical gears. The rattle threshold is determined by analyzing the structure-borne noise and angular acceleration levels as well as vibro-impacts in time domain. Increasing the excitation frequency influences the impact occurrence per revolution and leads to a decreased rattle threshold. After exceeding the rattle threshold, the structure-borne noise levels at different excitation frequencies applied converge and at a certain speed oscillation amplitude, the structure-borne noise levels are comparable. The rattle threshold is influenced by the drag torque - nevertheless, the structure-borne noise and angular acceleration levels at different drag torques applied do not deviate significantly after exceeding the rattle threshold. The lubrication shows the highest impact on gear rattling at the given test parameters, comparing the lubricants SAE 0W-16 and SAE $85 \mathrm{~W}-90$. The structure-borne noise indicates higher damping of SAE $85 \mathrm{~W}-90$ when analyzing the decay curve at constant speed oscillation amplitude. The damping effect of the lubricant decreases when increasing the excitation frequency at constant speed oscillation amplitude.
\end{abstract}

\section{Einleitung und Motivation}

Die Anforderungen an die Schwingungs- und Geräuschqualität von Automobilen haben sich durch die Gesetzgebung und den Wettbewerb in den letzten Jahrzehnten deutlich erhöht [1]: Der Geräuschpegel in der Fahrerkabine ist bei konstanter Fahrgeschwindigkeit durchschnittlich um ca. 1,5-2 dB(A) mit jeder neuen Automobilgeneration gesunken [1]. In der Automobilbranche werden jährlich Gewährleistungskosten für Störgeräusche im mehrstelligen Millionenbereich pro Großserienfahrzeug ausgegeben [2]. Aufgrund der tonalen oder rasselnden Geräuschcharakteristik werden Getriebegeräusche trotz niedrigem Geräuschpegel im Fahrgeräusch als störend wahrgenommen [1]. Neben dem tonalen Getriebeheulen ist das impulsartige Rasselgeräusch in der Fahrerkabine von hoher Bedeutung [2].

Das Rasseln tritt bei stirnradverzahnten Stufengetrieben infolge von Drehschwingungen des Verbrennungsmotors und aufgrund von aufgeprägten Torsionsschwingungen im Resonanzbereich des Triebstrangs auf [2]. Die resultierende Kraft aus den Gaskräften der diskontinuierlichen Verbrennungsprozesse und den Massenkräften unausgeglichener Motormassen ist der Kurbelwellendrehbewegung überlagert und führt zur Drehschwingung [2]. Beim Verbrennungsmotor wird die Kurbelwelle im Arbeitstakt beschleunigt und im Verdichtungstakt gebremst - die Kurbelwelle eines Viertaktmotors benötigt für diese Prozessabfolge zwei vollständige Rotationen [3]. Ein Vierzylinder-Viertaktmotor in Reihenmotorbauform generiert beispielsweise eine der Zündfrequenz des Verbrennungsmotors entsprechende Drehschwingung zweiter Ordnung [4]. Aufgrund steigender Zylinderdrücke infolge der Aufladung, weniger Zylin- der durch Downsizing, optimierten Verbrauchs und verminderten Ausstoßes von Abgasen werden Drehschwingungen im Antriebsstrang erhöht [2]. Insbesondere beim Aufeinandertreffen der Torsionseigenfrequenzen des Antriebsstrangs mit der induzierten Anregung des Verbrennungsmotors sind Rasselgeräusche deutlich wahrnehmbar [2].

Die experimentelle Untersuchung des Rasselgeräuschverhaltens von Getrieben kann durch die Verwendung eines antreibenden Elektromotors durchgeführt werden, welcher die Drehungleichförmigkeit eines Verbrennungsmotors abbilden kann [2]. Die kontinuierliche Steigerung der Drehzahlungleichförmigkeit führt bei einer mittleren Antriebsdrehzahl infolge der induzierten Torsionsschwingungen zu einer Schwingungsanregung der Losteile [2]. Die Drehungleichförmigkeit führt ab einer bestimmten Winkelbeschleunigungsamplitude zu einem Abheben der Zahnflanken (Abb. 1, Punkt 1) [5]. Nach dem Durchlauf des Verdrehflankenspiels (Punkt 2) stößt die Zahnflanke des getriebenen Rades impulsartig gegen die Schubflanke des treibenden Rades (Punkt 3) [5]. Nach der Haftphase (Punkt 4) löst sich das getriebene Rad (Punkt 5) und es kommt zu einem Verdrehflankenstoß auf der Zugflanke des treibenden Rades (Punkt 6) [5]. In der Literatur sind umfangreiche Untersuchungen verschiedener Einflussgrößen auf das Rasselgeräuschverhalten von Getriebevarianten auf Basis des emittierten Luftschalls dokumentiert. Eine fundierte Einflussgrößenanalyse auf die Rasselgeräuschneigung von einstufigen Stirnradgetrieben basierend auf Körperschall- und Drehbeschleunigungsmessungen ist kaum vorhanden. 
Abb. 1 Problemstellung und Lösungsansatz

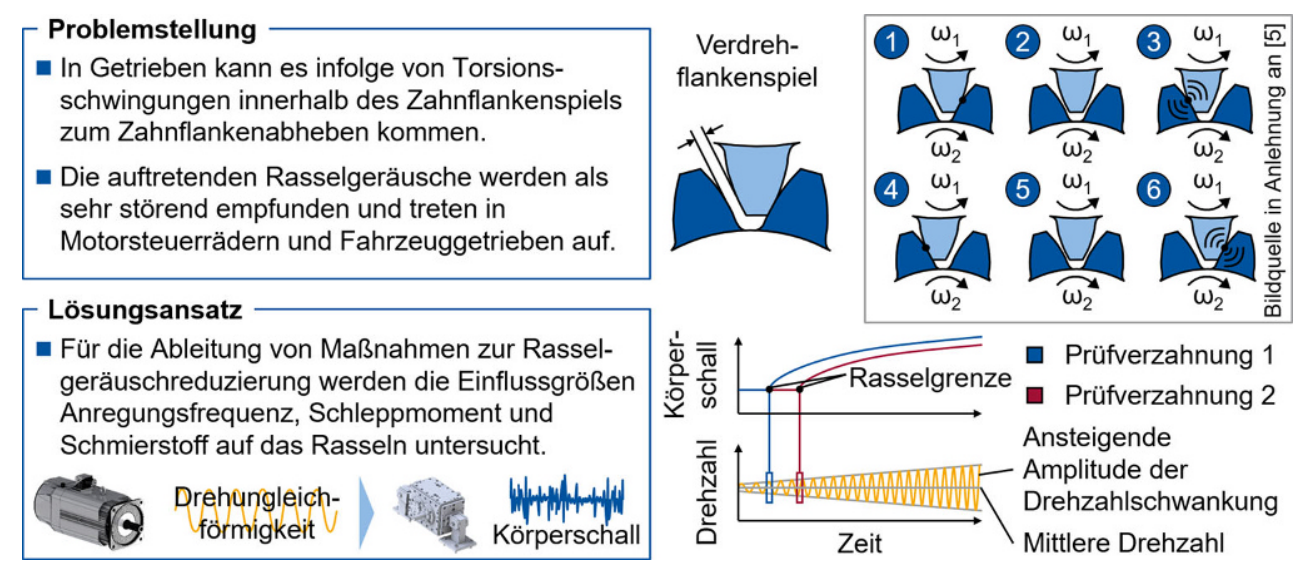

\section{Stand der Technik}

Die Rasselgeräusche werden neben dem Getriebeheulen als Komfortproblem in der Fahrerkabine betrachtet und eine alleinige Analyse des Gesamtgeräuschpegels ist unzureichend [2]. Neben subjektiver und objektiver Geräuschevaluierung werden bei Rasselgeräuschen die Stöße und Impulse im Zeit- und Frequenzbereich untersucht [2]. Das Rasselgeräusch ist breitbandig mit Frequenzen ab ca. $500 \mathrm{~Hz}$ bis mehr als $5 \mathrm{kHz}$ und moduliert mit der ersten oder zweiten Zündordnung [2, 6]. Weitere akustische Eigenschaften des Rasselgeräusches sind eine stochastische Signalart und eine hohe Modulationsfrequenz [7]. Eine Unterscheidung zwischen Klapper- und Rasselgeräuschen kann nach LANG [8] gemäß des Getriebe- und des Fahrzustandes erfolgen: Die Getriebegeräusche werden bei geschlossener Kupplung in Getriebeneutralstellung als Klappern und im Zug- oder Schubbetrieb bei eingelegtem Gang als Rasseln bezeichnet [9]. Nach JÖRG [10] können Rasselgeräusche anhand des Motorbetriebszustands in Leerlaufrasseln, Zugund Schubrasseln eingeteilt werden [9]. Die Klapper- und Rasselgeräusche treten bei Drehzahlen zwischen $0 \mathrm{~min}^{-1}$ und $2500 \mathrm{~min}^{-1}$ auf - bei größeren Drehzahlen reicht das Schleppmoment aus, ein Zahnflankenabheben zu verhindern [1]. Die Klapper- und Rasselgeräusche sind primär bei stirnradverzahnten Handschalt- und Doppelkupplungsgetrieben vorzufinden [1]. Aufgrund der geringen mittleren Belastung gegenüber der dynamischen Belastungen ist eine rasselnde Bewegung zudem bei Steuerrädern im Steuertrieb zu beobachten [11]. In der Literatur wird das generierte Geräusch bei Steuerrädern teilweise auch als Zahnradhämmern aufgrund der auftretenden kurzzeitigen und hohen Kontaktkräfte oder allgemein als Steuertriebsgeräusch bezeichnet $[11,12]$; der Entstehungsmechanismus ist aber ähnlich zum Rasseln und daher wird in diesem Bericht konsequent die Bezeichnung Rasseln verwendet.

\subsection{Einflussgrößen auf das Rasselgeräuschverhalten in Fahrzeuggetrieben}

Die experimentelle Untersuchung der Auswirkungen des Abhebens und des erneuten Aufeinandertreffens der Zahnflanken in Getrieben ist in einigen Arbeiten durchgeführt worden. WECK ET AL. untersuchen die Auswirkungen des Zahnflankenabhebens bedingt durch fremderregte, niederfrequente Anregungen auf die Geräuschemission und die Zahnflankenbeanspruchung in Getrieben [13]. Hierzu wird die Fremdanregung durch den Antrieb eines Verspannungsprüfstands mit einer Doppelgelenkwelle mit versetzten Gelenken hervorgerufen und die Zusatzbeanspruchung der Zahnflanken infolge des Aufpralls durch Zahnfußdehnungsmessungen nachgewiesen [13]. Der Einfluss des Verspannmoments wird bei $M_{v}=50 \mathrm{Nm}$ und $M_{v}=100 \mathrm{Nm}$, der Einfluss des Zahnflankenspiels wird bei $\mathrm{j}=0,2 \mathrm{~mm}$ und $\mathrm{j}=0,4 \mathrm{~mm}$ und der Einfluss der Schmierstoffviskosität wird bei $v_{50}{ }^{\circ} \mathrm{C}=60 \mathrm{~mm}^{2} / \mathrm{s}$ und $v_{40}{ }^{\circ} \mathrm{C}=320 \mathrm{~mm}^{2} / \mathrm{s}$ untersucht [13]. Der Versatzwinkel wird zwischen $\beta_{\mathrm{V}}=0^{\circ}$ und $\beta_{\mathrm{V}}=25^{\circ}$ variiert [13]. Das Verspannmoment (ca. $\Delta \mathrm{L}=5 \mathrm{~dB}$ ), die Schmierstoffviskosität (ca. $\Delta \mathrm{L}=5 \mathrm{~dB}$ ) und der Versatzwinkel (ca. $\Delta \mathrm{L}=13 \mathrm{~dB}$ ) haben den größten Einfluss auf den gemessenen Körperschallsummenpegel für das gewählte Prüfkonzept, während der Einfluss des Zahnflankenspiels gering ist (ca. $\Delta \mathrm{L}=1 \mathrm{~dB}$ ) [13]. Auch Motorsteuerräder neigen zu einer rasselnden Bewegung und es kommt zur Geräuschemission infolge von Zahnflankenstößen. WILHELM ET AL. untersuchen den Steuertrieb von zwei unterschiedlichen Dieselmotoren mit drei Zylindern durch Körperschallmessungen an den Lagerstellen der Zahnräder und der Wellen sowie durch die Messung der Tangentialbeschleunigungen an den Zahnrädern und stellen fest, dass die periodischen Anregungen bezogen auf den Motorzyklus gegenüber den stochastischen Anregungen dominieren [14]. TATAR ET AL. untersuchen das Rasselgeräuschverhalten und das Getriebeheulen des Steuertriebs eines 12,7-Liter-Schwerlast-Dieselmotors bei einer 
Abb. 2 Einflussgrößen auf das Rasselgeräuschverhalten $[3,5,18]$. Bildquelle: [19]

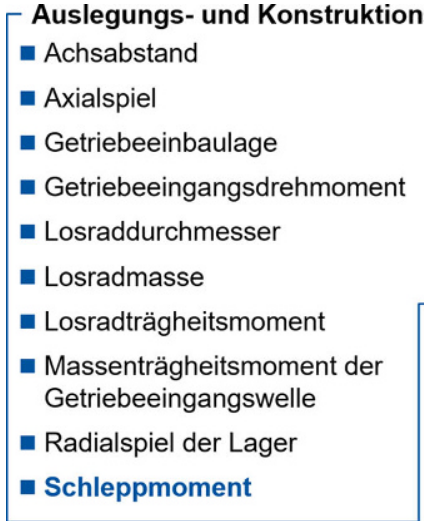

parameter

- Schmierstoff (Additive, Viskosität)

- Schrägungswinkel

- Übersetzungsverhältnis

- Verdrehflankenspiel

- Verzahnungsoberfläche

Werkstoff

- Betriebsparameter

- Anregungsfrequenz

- Grunddrehzahl

- Winkelbeschleunigungsamplitude

\begin{tabular}{l} 
Anregungsfrequenz \\
Variation der Anzahl \\
an Sinusschwingung- \\
en pro Umdrehung \\
\hline Schleppmoment \\
Variation der axialen \\
Lagervorspannungs- \\
kraft \\
\hline $\begin{array}{l}\text { Schmierstoff } \\
\text { Variation } \\
\text { der Schmierstoff- } \\
\text { spezifikation }\end{array}$ \\
\hline
\end{tabular}

Drehzahl von $n=550 \mathrm{~min}^{-1}$ durch Luftschalldruckmessungen [15]. Die experimentellen Untersuchungen zeigen eine Reduktion des Rasselgeräuschverhaltens und ein Anheben des Getriebeheulens infolge der Zahnradbelastung durch eine Servopumpe und die maximale Luftschalldruckpegeldifferenz beträgt $\Delta \mathrm{L}=1,8 \mathrm{~dB}(\mathrm{~A})$ zwischen belasteten und lastfreien Zustand [15]. RACH untersucht verschiedene Einflussgrößen auf das Rasselgeräuschverhalten eines Front-Quer-Getriebes und eines Koaxialgetriebes und leitet Konstruktionsempfehlungen für rasselgeräuschreduzierte Getriebe ab [16]. DoĞAN untersucht experimentell verschiedene Einflüsse auf das Rasselgeräuschverhalten von PKW-Getrieben mittels Luftschalldruckmessungen [5]. Zusätzlich wird eine Luftschalldruckpegeldifferenz in Abhängigkeit des Verdrehflankenspiels (ca. $\Delta \mathrm{L}=6 \mathrm{~dB}(\mathrm{~A})$ ), des Losradaxialspiels (ca. $\Delta \mathrm{L}=4 \mathrm{~dB}(\mathrm{~A})$ ), des Losradradialspiels (ca. $\triangle \mathrm{L}=5 \mathrm{~dB}(\mathrm{~A})$ ) und der Verzahnungsoberfläche (ca. $\Delta \mathrm{L}=3 \mathrm{~dB}(\mathrm{~A})$ ) lastfrei an einer Gangstufe in einem Prüfgetriebe mit stehender Abtriebswelle bei einer mittleren Drehzahl von $n=800 \mathrm{~min}^{-1}$ festgestellt [5]. Der Einfluss des Verdrehflankenspiels wird für die Verdrehflankenspiele $\mathrm{j}=0,06 \mathrm{~mm}, \mathrm{j}=0,12 \mathrm{~mm}$ und $\mathrm{j}=0,18 \mathrm{~mm}$ bei einer Winkelbeschleunigungsamplitude von $\Delta \omega=600 \mathrm{rad} / \mathrm{s}^{2}$ analysiert [5]. Der Einfluss des Losradaxialspiels wird zwischen den Grenzen $s_{a}=0,1 \mathrm{~mm}$ und $s_{a}=0,4 \mathrm{~mm}$ bei einer Winkelbeschleunigungsamplitude von $\Delta \omega=600 \mathrm{rad} / \mathrm{s}^{2}$ untersucht [5]. Der Einfluss des Losradradialspiels wird bei $\mathrm{s}_{\mathrm{r}}=0,03 \mathrm{~mm}, \mathrm{~s}_{\mathrm{r}}=0,10 \mathrm{~mm}$ und $\mathrm{s}_{\mathrm{r}}=0,20 \mathrm{~mm}$ für verschiedene Winkelbeschleunigungsamplituden bis $\Delta \omega=3000 \mathrm{rad} / \mathrm{s}^{2}$ untersucht [5]. Der Einfluss der Verzahnungsoberfläche wird durch einen Vergleich zwischen geschliffener und beschichteter Topografie für verschiedene Winkelbeschleunigungsamplituden bis $\mathrm{zu} \Delta \omega=3000 \mathrm{rad} / \mathrm{s}^{2}$ analysiert [5]. STOCKMEIER entwickelt ein klapper- und rasselgeräuschfreies Getriebe (KURF-Getriebe) und zeigt das Potenzial der Festradentkopplung anhand einer Zahnradstufe auf, wobei eine Luftschalldruckpegelreduzierung von mehr als $\Delta \mathrm{L}=20 \mathrm{~dB}(\mathrm{~A})$ zwischen gekoppeltem und entkoppeltem Zustand gezeigt wird [3]. NOVAK konstruiert ein klapper- und rasselgeräuschfreies Getriebe (KURFGetriebe) mittels einer Festradentkopplung und vergleicht das Rasselgeräuschverhalten mit einem nachgebildeten Serienradsatz in einem Prüfgetriebe, wobei eine Luftschalldruckpegeldifferenz von $\Delta \mathrm{L}=15,5 \mathrm{~dB}(\mathrm{~A})$ bei einer mittleren Drehzahl von $n=800 \mathrm{~min}^{-1}$, einer Winkelbeschleunigungsamplitude von $\Delta \omega=4000 \mathrm{rad} / \mathrm{s}^{2}$ und einer Betriebstemperatur von $\mathrm{T}=80^{\circ} \mathrm{C}$ erzielt wird [17]. BAUMANN untersucht den Einfluss der Schmierstoffzusammensetzung auf das Rasselgeräuschverhalten durch Variation der Grundöle und der Additivierung mittels Luftschalldruckmessungen an einem Handschaltgetriebe [9]. Der Luftschalldruckpegel wird durch ein wasserlösliches Polyalkylenglykol um $\Delta \mathrm{L}=4 \mathrm{~dB}(\mathrm{~A})$ im Vergleich zur Ölbefüllung in der Serie bei fast gleicher Schmierstoffviskosität gesenkt [9]. Das Grundöl ist jedoch aufgrund der Begünstigung von Korrosion für die Anwendung in Fahrzeuggetrieben nicht geeignet [9]. FIETKAU entwickelt ein Modell zur Bestimmung des Rasselgeräusches und verifiziert das Modell mittels Rasselgeräuschuntersuchungen an einem Prüfgetriebe bei einer mittleren Drehzahl von $n=900 \mathrm{~min}^{-1}$ durch Körperschall-, Luftschalldruck- und Differenzwinkelmessungen [4]. Der Einfluss des Verdrehflankenspiels auf den gemessenen Körperschallpegel am Gehäuse beträgt bei den Verdrehflankenspielen $j=0,081 \mathrm{~mm}, j=0,189 \mathrm{~mm}$ und $\mathrm{j}=0,374 \mathrm{~mm}$ bis $\mathrm{zu} \Delta \mathrm{L}=6 \mathrm{~dB}$ in einem Bereich der Winkelbeschleunigungsamplituden von $\Delta \omega=0 \mathrm{rad} / \mathrm{s}^{2}$ bis $\Delta \omega=1000 \mathrm{rad} / \mathrm{s}^{2}$ [4]. DION ET AL. analysieren den Zahnflankenstoß experimentell zwischen zwei Zahnflanken mittels externer Anregung durch einen elektrodynamischen Schwingungserreger mit einer maximalen Anregungsamplitude von $\Delta \omega=2000 \mathrm{rad} / \mathrm{s}^{2}$ und einem Abtriebsrad als Losrad [6]. Die Untersuchungen umfassen eine Parameterstudie zwischen den Anregungsfrequenzen $\mathrm{f}=30 \mathrm{~Hz}$ und $\mathrm{f}=60 \mathrm{~Hz}$ jeweils bei konstanter Weg-, Geschwindigkeitsund Beschleunigungsamplitude und zeigen die Auswirkung auf die Anzahl und das Energieniveau der Zahnflankenstöße [6]. Die Zahnflankenstöße erfolgen unter bestimmten Anregungszuständen zunächst mehrfach auf einer Zahn- 


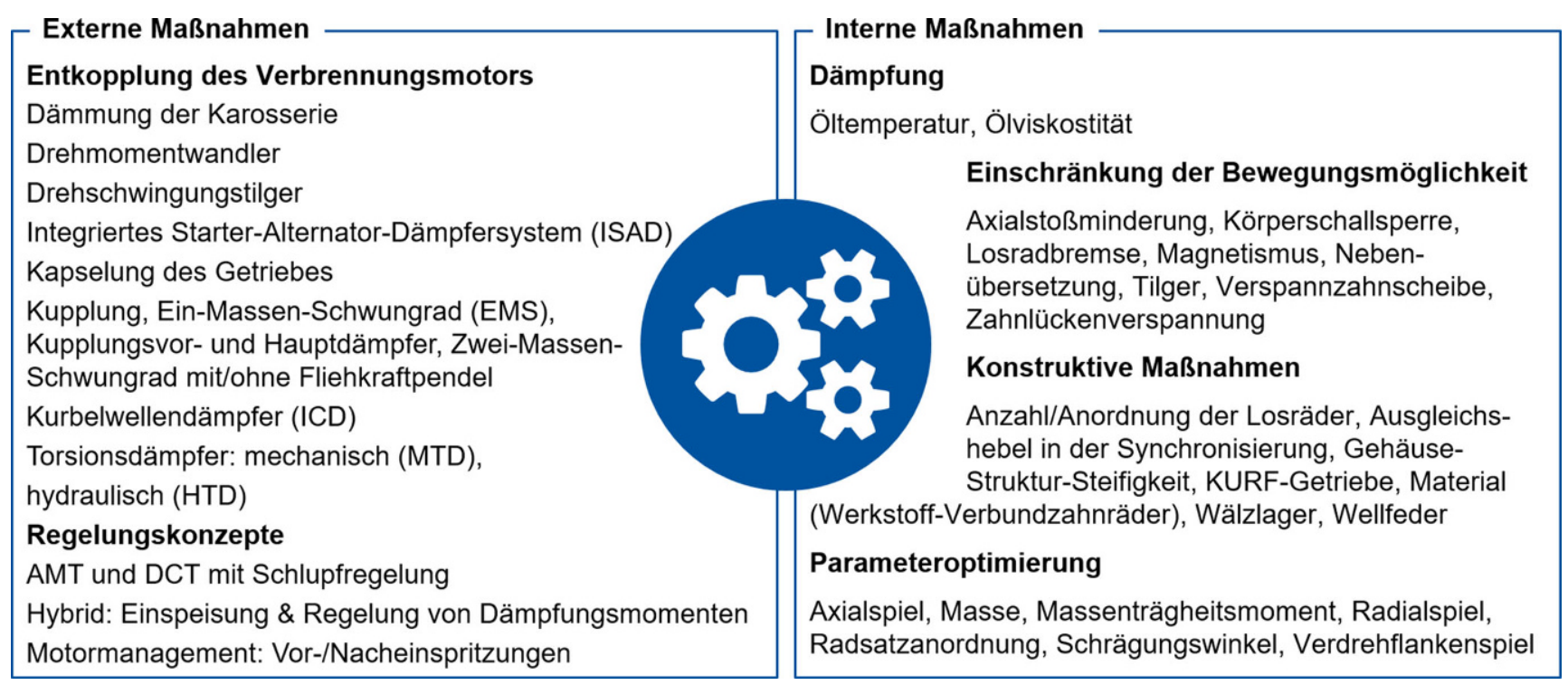

Abb. 3 Externe und interne Maßnahmen zur Klapper- und Rasselgeräuschreduzierung [2, 3, 9, 17, 18]

flanke und bei einer Erhöhung der Anregungsintensität auf beiden Zahnflanken [6].

Die Einflussgrößen auf das Rasselgeräuschverhalten können in Auslegungs- und Konstruktionsparameter sowie in Betriebsparameter unterteilt werden (Abb. 2). Die Konstruktionsparameter beinhalten das Axialspiel, den Losraddurchmesser, die Losradmasse, das Losradträgheitsmoment, das Radialspiel der Lager, das Schleppmoment, den Schmierstoff, den Schrägungswinkel, das Übersetzungsverhältnis, das Verdrehflankenspiel sowie den Werkstoff. Die chemische Zusammensetzung und die kinematische Viskosität des Schmierstoffs beeinflussen den Schmierfilmaufbau sowie das Schleppmoment und somit das Rasselgeräuschverhalten signifikant [9]. Der Reibwert ist beispielsweise von der Grundflüssigkeit und der Additivierung des Schmierstoffs abhängig und eine Verringerung des Reibwerts ist bezüglich eines verringerten Rasselgeräuschs und eines verringerten Verlustmoments vorteilhaft, da sich sowohl die Energie zum Scheren des Schmierstofffilms als auch die Kraftübertragung durch den Schmierstoff verringern [9]. Die relevanten Auslegungsparameter umfassen bei Koaxial-Getrieben den Achsabstand, das Getriebeeingangsdrehmoment und das auf die Getriebeeingangswelle reduzierte Massenträgheitsmoment [18]. Zusätzlich hat die Getriebeeinbaulage einen Einfluss auf das Rasselgeräuschverhalten, da sie die Anzahl an in Schmierstoff eingetauchten Losteilen durch Rotation des Getriebes um die Fahrzeuglängsachse bestimmt [5]. Die Struktur der Verzahnungsoberfläche wirkt sich auf die Dämpfung der Zahnflankenstöße aus - infolge einer besseren Schmierstoffhaftung durch eine strukturierte Verzahnungsoberfläche wird beispielsweise die Dämpfung beim Aufeinandertreffen der Zahnflanken gesteigert [5].
Der Betrag des Schleppmoments $T_{S}$ setzt sich aus dem Lagerreibmoment $T_{L}$, dem Planschmoment $T_{P l}$, dem Quetschmoment $T_{Q u}$ und dem Synchronisierungsschleppmoment $T_{S y}$ zusammen [20]:

$T_{S}=T_{L}+T_{P l}+T_{Q u}+T_{S y}$

$T_{S} \quad[\mathrm{Nm}] ;$ Schleppmoment

$T_{L} \quad[\mathrm{Nm}]$; Lagerreibmoment

$T_{P l}[\mathrm{Nm}] ;$ Planschmoment

$T_{Q u}[\mathrm{Nm}] ;$ Quetschmoment

$T_{S y} \quad[\mathrm{Nm}] ;$ Synchronisierungsschleppmoment

Das Lagerreib- und das Synchronisierungsschleppmoment werden von der Relativdrehzahl des Losrades zur Welle beeinflusst [20]. Das Planschmoment tritt beim Eintauchen des Losrades in das Schmierstoffreservoir auf und ist von der Winkelgeschwindigkeit des Losrades abhängig [20]. Das Quetschmoment resultiert aus der Schmierstoffverdrängung im Zahneingriff [20].

Das gesamte Reibungsmoment $\mathrm{T}_{\mathrm{L}}$ eines Wälzlagers ist die Addition aus dem lastunabhängigen Reibungsmoment $\mathrm{T}_{0}$ und dem lastabhängigen Reibungsmoment $\mathrm{T}_{1}$ [21]:

$T_{L}=T_{0}+T_{1}$

$T_{L} \quad$ [Nm]; Gesamtes Lagerreibungsmoment

$T_{0} \quad$ [Nm]; Lastunabhängiges Reibungsmoment

$T_{1} \quad[\mathrm{Nm}]$; Lastabhängiges Reibungsmoment 
Abb. 4 Zielsetzung und Vorgehensweise

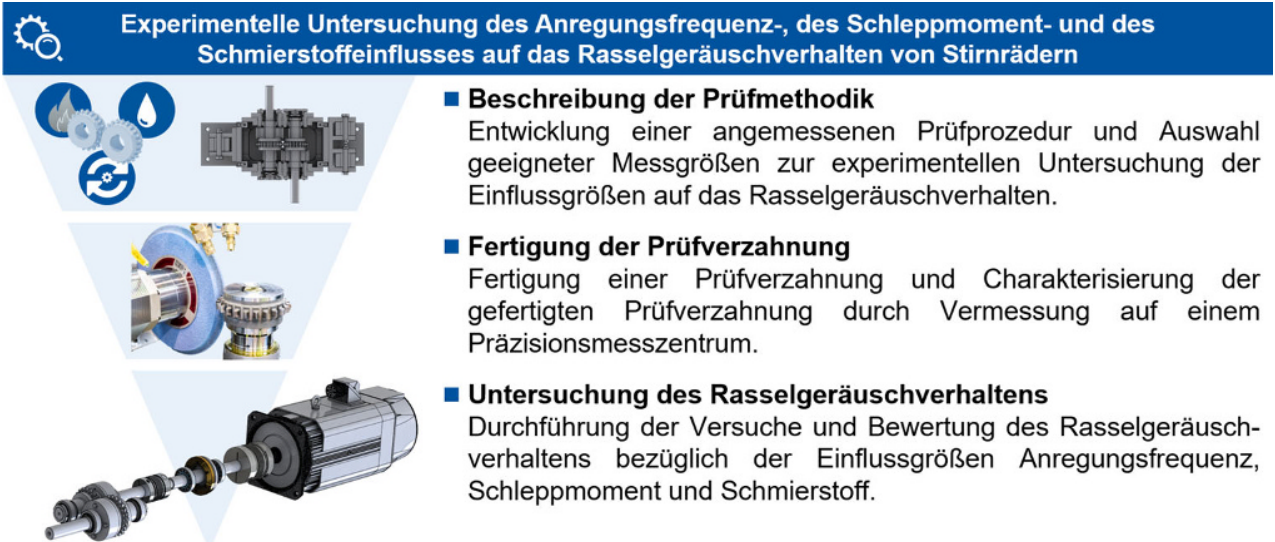

- Beschreibung der Prüfmethodik

Entwicklung einer angemessenen Prüfprozedur und Auswahl geeigneter Messgrößen zur experimentellen Untersuchung der Einflussgrößen auf das Rasselgeräuschverhalten.

Fertigung einer Prüfverzahnung und Charakterisierung der gefertigten Prüfverzahnung durch Vermessung auf einem .

Durchführung der Versuche und Bewertung des RasselgeräuschSchleppmoment und Schmierstoff.

Das lastunabhängige Reibmoment $\mathrm{T}_{0}$ wird durch die temperaturabhängige Schmierstoffviskosität im Betrieb, die Betriebsdrehzahl, die Lagerbauart, die Lagergröße und die Schmierungsart bestimmt [21]. Das lastabhängige Reibungsmoment $T_{1}$ ist hingegen nur geringfügig von der Betriebsdrehzahl abhängig - unter der Annahme vollständig getrennter Oberflächen durch den Schmierfilm - und durch die Lagerbelastung, -bauart und -größe festgelegt [21]. Das Schleppmoment wird in diesem Bericht durch das Einstellen unterschiedlicher Lagervorspannungskräfte für die angestellte Kegelrollenlagerung variiert, welche den lastabhängigen Reibungsmomentanteil beeinflussen.

\subsection{Maßnahmen zur Rasselgeräuschreduzierung}

Dem Klapper- und Rasselgeräuschverhalten von Getrieben wird anhand von internen und externen Maßnahmen begegnet (Abb. 3). Die externen Maßnahmen basieren auf der Verminderung von Torsionsschwingungen außerhalb des Getriebes [18]. Der Fokus liegt insbesondere bei der Abkopplung der Motoranregung vom Antriebsstrang [18]. Die Regelungskonzepte umfassen das Motormanagement zur Reduzierung der Drehungleichförmigkeit der Kurbelwelle, die Schlupfregelung bei automatischer Betätigung der Kupplung zum Abbauen von Drehschwingungsüberhöhungen sowie die Schwingungsdämpfung durch die Einleitung und Regelung von Dämpfungsmomenten mit dem E-Motor in einem hybridisierten Antriebsstrang [9]. Die internen Maßnahmen basieren auf der Dämpfung, der Einschränkung der Bewegungsmöglichkeit von Losteilen, konstruktive Maßnahmen sowie der Parameteroptimierung. Aufgrund der Einschränkung von Losteilen innerhalb ihres funktionsbedingten Spiels kann das Auftreten von Stößen unterdrückt oder abgeschwächt werden [18]. In [3] wird ein klapper- und rasselgeräuschfreies Getriebe (KURFGetriebe) entwickelt, welches durch Festradentkopplung der nicht am Leistungsfluss beteiligten Zahnräder die Einleitung der Drehungleichförmigkeit des Verbrennungsmo- tors in das Getriebe unterbricht [17]. Den Vorteilen eines KURF-Getriebes, wie die Reduzierung von Geräuschen und Schleppmomenten sowie zusätzliche Möglichkeiten bei der Schaltstrategie, stehen die Nachteile der steigenden Komplexität, der erhöhten Bauteilanzahl sowie der Gewichts- und Kostenzunahme entgegen [3].

\section{Zielsetzung und Vorgehensweise}

In der Literatur wird die Rasselgeräuschneigung von Getriebevarianten aufgrund des emittierten Luftschalls miteinander verglichen. Infolge des Transferpfadeinflusses sind Körperschallmessungen für die Evaluierung des Rasselgeräuschverhaltens an ganzheitlichen Getrieben wegen der unterschiedlichen Getriebegehäuse nicht aussagekräftig. Die Untersuchung der Einflussgrößen auf das Rasselgeräuschverhalten einzelner Radsätze ohne den Einfluss der Geräuschquellen weiterer Losteile ist in geringem Umfang dokumentiert. Eine fundierte Einflussgrößenanalyse auf das Rasselgeräuschverhalten von einstufigen Stirnradgetrieben basierend auf Körperschallmessungen am Getriebegehäuse und Drehbeschleunigungsmessungen nahe dem Verzahnungseingriff mit einer gezielten Variation des Schleppmoments ist nicht vorhanden. Das Ziel des Berichts ist daher die experimentelle Untersuchung der Einflussgrößen Anregungsfrequenz, Schleppmoment und Schmierstoffspezifikation auf das Rasselgeräuschverhalten von Stirnrädern. Das Vorhaben wird in drei Teilschritte aufgeteilt (Abb. 4).

Der erste Schritt umfasst die Beschreibung der Prüfverzahnung und des Prüfstandsaufbaus. Zudem werden eine fähige Prüfprozedur entwickelt und die Messgrößen definiert, um die Einflussgrößen Anregungsfrequenz, Schleppmoment und Schmierstoff auf das Rasselgeräuschverhalten zu untersuchen. In der Prüfprozedur werden die Anregungsfrequenzen, die Lagervorspannungskräfte, die mittleren Drehzahlen und die Schwankungsamplituden der aufzuprägenden Drehzahlschwankungen festgelegt, welche durch 
Abb. 5 Verzahnungsdaten der Prüfverzahnung

$$
\begin{aligned}
& \text { Makrogeometrie } \\
& \square \mathrm{m}_{\mathrm{n}}=5 \mathrm{~mm} \\
& \square \mathrm{z}_{1 / 2}=21 / 23 \\
& \square \mathrm{a}=112,5 \mathrm{~mm} \\
& \square \mathrm{a}_{\mathrm{n}}=20^{\circ} \\
& \square \beta_{1 / 2}=0^{\circ} \\
& \square \mathrm{b}=10 \mathrm{~mm} \\
& \square \mathrm{x}_{1 / 2}=0,280 \\
& \square \varepsilon_{\alpha / \beta / \mathrm{v}}=1,47 / 0 / 1,47
\end{aligned}
$$

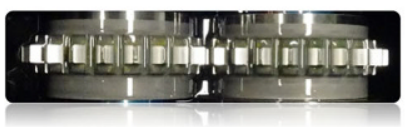

Mikrogeometrie

- $\mathrm{C}_{\mathrm{a}, 1 / 2}=0 / 0 \mu \mathrm{m}$

- $\mathrm{C}_{\beta, 1 / 2}=2 / 8 \mu \mathrm{m}$

- $\mathrm{f}_{\mathrm{h \alpha}, 1 / 2}=0 / 0 \mu \mathrm{m}$

- $\mathrm{f}_{\mathrm{h} \beta, 1 / 2}=0 / 0 \mu \mathrm{m}$

- $\mathrm{C}_{\mathrm{a}, 1 / 2}=90 / 90 \mu \mathrm{m}$ zirkula

- $\mathrm{d}_{\mathrm{Ca}, 1 / 2}=114,3 / 124,2 \mathrm{~mm}$$$
4
$$

. ilintir
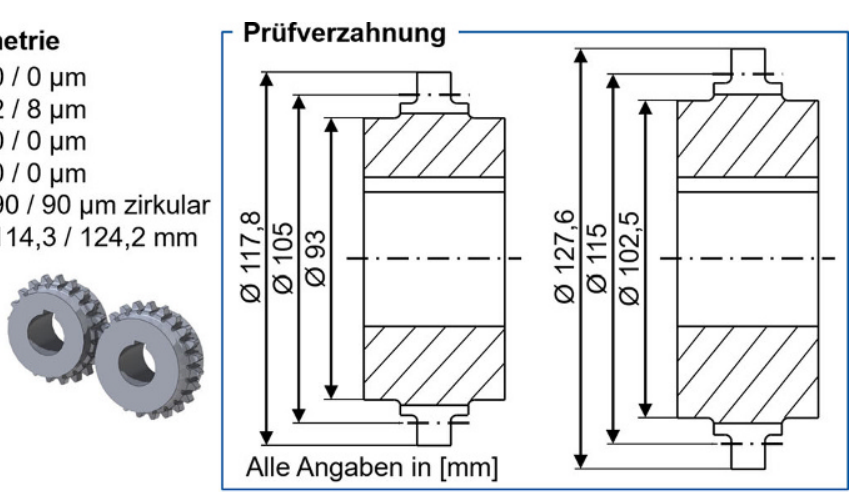

einen elektrischen Antriebsmotor vorgegeben werden und die Prüfverzahnung zum Rasseln anregen. Die Anregungsfrequenz kann durch die Regelung des elektrischen Antriebmotors variiert werden. Die Variation des Schleppmoments wird durch das Einstellen unterschiedlicher Lagervorspannungskräfte realisiert. Zwischen den Messpunkten gleicher aufgeprägter Drehzahlschwankung und unterschiedlicher Lagervorspannungskraft wird ein vergleichbares Temperaturniveau angestrebt, um den Einfluss der temperaturabhängigen Schmierstoffviskosität gering zu halten. Der Messzyklus wird für zwei Schmierstoffspezifikationen unterschiedlicher Viskosität durchgeführt. Im zweiten Schritt wird eine Prüfverzahnung auf einer Höfler StirnradSchleifmaschine Viper 500 KW [22] geschliffen. Die Prüfverzahnung wird anschließend auf einem Präzisionsmesszentrum P16 der Firma Klingelnberg [23] vermessen und charakterisiert. Im dritten Schritt erfolgt die Versuchsdurchführung auf dem Rasselgeräuschprüfstand. Die Versuchsergebnisse werden analysiert und das Rasselgeräuschverhalten wird bezüglich der Einflussgrößen Anregungsfrequenz, Schleppmoment und Schmierstoff bewertet.

\section{Prüfmethodik zur Untersuchung des Rasselgeräuschverhaltens}

Zunächst wird die Prüfverzahnung vorgestellt. Anschließend werden der Prüfstandsaufbau und die verwendete Messtechnik beschrieben. Am Ende des Kapitels wird die Prüfprozedur mit den unterschiedlichen Versuchsparametern erläutert.

\subsection{Prüfverzahnung}

Die Prüfverzahnung ist eine geradverzahnte Stirnradverzahnung mit einem Modul von $\mathrm{m}_{\mathrm{n}}=5 \mathrm{~mm}$. Die Prüfverzahnung wird mittels eines bestehenden Prüfkonzepts in Anlehnung an CARL [24] untersucht, nach welchem gerad- und schrägverzahnte Stirnräder getestet werden können. Die Daten der Makro- und der Mikrogeometrie können der Abb. 5 ent- nommen werden. Zudem ist eine zirkulare Kopfrücknahme realisiert, um die Gefahr eines vorzeitigen Zahneingriffsstoßes zu verringern. Die Profilüberdeckung der geradverzahnten Prüfverzahnung beträgt $\varepsilon_{\alpha}=1,47$.

\subsection{Prüfstandsaufbau}

Die Prüfverzahnung wird in ein Prüfgetriebe in Anlehnung an CARL [24] eingebaut, welches in den Triebstrang eines Rasselprüfstands integriert wird. Der Prüfstandsaufbau und die Messstellen für die Erfassung der Messgrößen können der Abb. 6 entnommen werden. Der Rasselprüfstand besitzt eine elektrische Antriebsmaschine mit einer Nennleistung $\mathrm{P}_{\mathrm{N}}=23,0 \mathrm{~kW}$ und einer maximalen Antriebsdrehzahl $\mathrm{n}_{1, \max }=7500 \mathrm{~min}^{-1}$. Die Regelung des Rasselprüfstands ermöglicht die Vorgabe von sinusförmigen Drehzahlschwankungen, welche die Prüfverzahnung zu Schwingungen anregen und zu einem rasselnden Betriebszustand führen. Neben der mittleren Drehzahl können die Anzahl an Sinusschwingungen pro Umdrehung und die Schwankungsamplitude der Drehzahl vorgegeben werden. Bei einem Viertaktmotor in Reihenmotorbauform ergibt sich beispielsweise die Motorordnung oder die Zündfrequenz aus dem Produkt aus der Zylinderanzahl und dem Faktor 0,5 [2]; eine Drehzahlschwankung mit 1,5-facher Sinusschwingung pro Umdrehung entspricht demnach einem Dreizylindermotor, eine 2-fache Sinusschwingung einem Vierzylindermotor, eine 2,5-fache Sinusschwingung einem Fünfylindermotor und eine 3-fache Sinusschwingung einem Sechszylindermotor. Zudem kann die Anregung der Prüfverzahnung entweder durch die Aufprägung einer Drehzahlschwankung mit konstanter Schwankungsamplitude oder mit linear ansteigender Schwankungsamplitude über die Anregungsdauer erfolgen. Die Größe der Drehzahlschwankungsamplitude entspricht der Größe der Drehzahlungleichförmigkeit und die Ableitung ergibt die maximale Drehbeschleunigung. Eine Zwischenwelle mit angeflanschtem Drehgeber überträgt die Drehzahlschwankung torsionssteif vom Antrieb auf die Antriebswelle des Prüfgetriebes durch die Anbindung mittels Metallbalgkupplungen. Das Prüfgetriebe nach CARL 
Abb. 6 Prüfstandsaufbau und Messgrößen
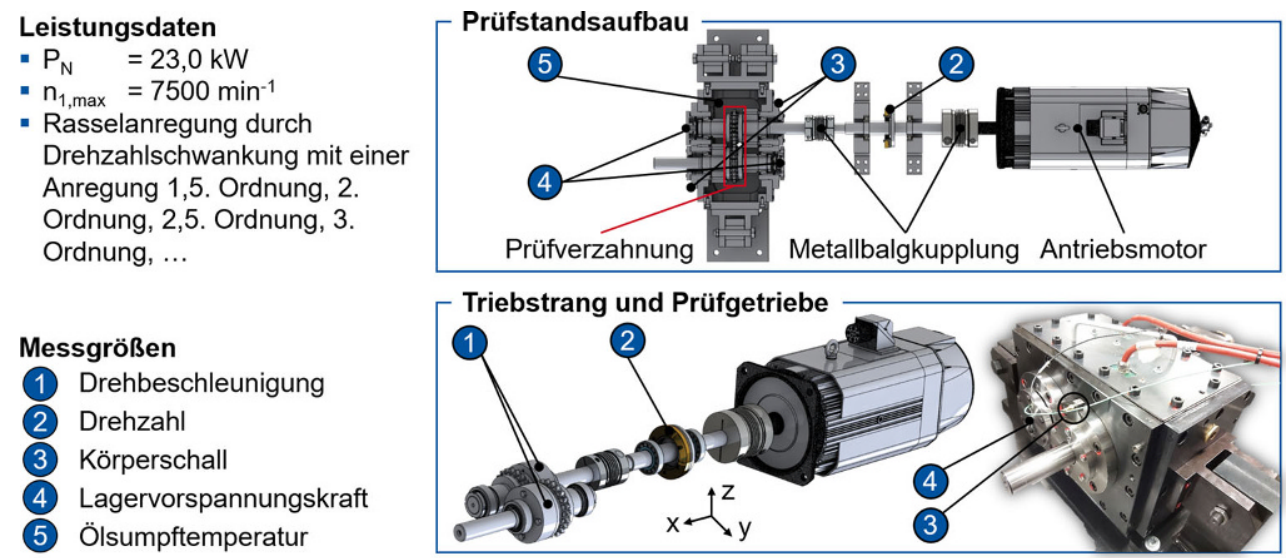

[24] ermöglicht die Untersuchung der Drehbeschleunigungen am Rad und am Ritzel anhand von Drehbeschleunigungsmesssystemen, die in unmittelbarer Nähe der Verzahnung für eine ausreichende messtechnische Erfassung der Radsatzanregung montiert werden. Die Schmierstoffversorgung der Prüfverzahnung erfolgt mittels einer Tauchschmierung, bei der die Prüfverzahnung um die Hälfte ihres Volumens in das Ölbad eintaucht. Das Wellen-Lager-System besteht aus Kegelrollenlager, die in angestellter X-Anordnung vorliegen [24].

\subsection{Messtechnik}

Die messtechnische Erfassung der Messgrößen während der Untersuchung des Rasselgeräuschverhaltens der Prüfverzahnung erfolgt anhand unterschiedlicher Sensoren und Messsysteme (Abb. 7). Die Drehbeschleunigungen $\ddot{\varphi}_{1}$ und $\ddot{\varphi}_{2}$ (1) werden am An- und Abtrieb der Prüfverzahnung mit einem 1-Kanal Telemetrie-System von Datatel detektiert. Nach HESSE ist die messtechnische Erfassung der Drehbeschleunigung möglichst nahe dem Zahneingriff für eine ausreichende Abbildung der Schwingungsanregung zu realisieren [25]. Das Messsystem besteht aus einem Telemetrie-Senderträger, der auf der Welle montiert ist, und einem am Gehäusedeckel angebrachten Telemetrie-Empfänger. Die rotierenden Komponenten des Messsystems werden induktiv gespeist und die berührungslose Übertragung der Messdaten zwischen dem rotierenden Telemetrie-Senderträger und dem stationären Telemetrie-Empfänger erfolgt anhand eines Antennensystems. Der Telemetrie-Senderträger besitzt zwei Beschleunigungssensoren, die um $180^{\circ}$ versetzt auf dem Messkreis liegen und die Drehbeschleunigung aufnehmen. Die Addition der zwei Beschleunigungssignale kompensiert die Erfassung radialer Schwingungen [24]. Die Erfassung der Drehzahl $\mathrm{n}_{1}$ (2) des elektrischen Antriebsmotors erfolgt mittels eines Heidenhain Drehgebers ERA 4282C mit 20.000 Inkrementen. Ein optischer Abtastkopf erfasst mit einer fotoelektrischen Abtas-

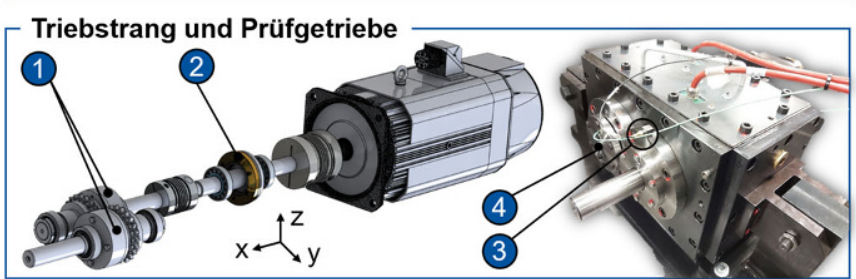

tung die Teilungen der Teilungstrommel, die auf dem Aufnahmeadapter der Welle montiert wird. Durch die fotoelektrische Abtastung werden die Teilungsstriche auf der Teilungstrommel, die mit der Welle rotiert, berührungslos detektiert. Das Rasselgeräuschverhalten wird anhand des Körperschalls a (3) ausgewertet, der mittels triaxialen Beschleunigungssensoren der Firma Brüel \& Kjær erfasst wird. Die triaxialen Beschleunigungssensoren werden auf den Lagerbuchsen am An- und Abtrieb des Prüfgetriebes adhäsiv mittels Zwei-Komponenten-Kleber befestigt. Die Messung der axialen Lagervorspannungskraft $\mathrm{F}_{\mathrm{Vor}}(4)$ wird durch piezoelektrische Kraftsensoren im Kraftfluss der Lagervorspannung am An- und Abtrieb realisiert. Die Ölsumpftemperatur $\mathrm{T}_{\text {Öl }}$ (5) innerhalb des Getriebes wird mit einem PlatinWiderstandsthermometer PT100 aufgenommen.

\subsection{Prüfprozedur}

Die Prüfprozedur ermöglicht die experimentelle Untersuchung der Einflussgrößen Anregungsfrequenz, Schleppmoment und Schmierstoffspezifikation auf das Rasselgeräuschverhalten. Zuerst wird das Tragbild des Radsatzes mittels Tragbildpaste analysiert, um eine angemessene Einbaulage von Ritzel und Rad zueinander zu gewährleisten. Im nächsten Schritt wird die axiale Lagervorspannungskraft $\mathrm{F}_{\mathrm{Vor}}$ der angestellten Kegelrollenlagerung separat für die An- und die Abtriebswelle eingestellt. Die axialen Lagervorspannungskräfte von $F_{\text {Vor }}=0,4 \mathrm{kN}, F_{\text {Vor }}=0,8 \mathrm{kN}$ und $\mathrm{F}_{\mathrm{Vor}}=1,2 \mathrm{kN}$ werden sowohl durch einen definierten Spaltabstand zwischen Lagerbuchsen und -deckeln als auch durch ein definiertes Anzugsdrehmoment der Lagerdeckelschrauben eingestellt und mittels der piezoelektrischen Kraftsensoren gemessen. Insgesamt werden die drei axialen Lagervorspannungskräfte jeweils für beide Schmierstoffspezifikationen SAE $0 \mathrm{~W}-16\left(v_{40}{ }^{\circ} \mathrm{C}=38,36 \mathrm{~mm}^{2} / \mathrm{s}\right)$ und SAE $85 \mathrm{~W}-90\left(v_{40}{ }^{\circ} \mathrm{C}=185 \mathrm{~mm}^{2} / \mathrm{s}\right)$ auf das Rasselgeräuschverhalten untersucht, sodass sich sechs Versuchsreihen ergeben (Abb. 8). Nach der Einstellung der Lagervorspannungs- 
Abb. 7 Messtechnik

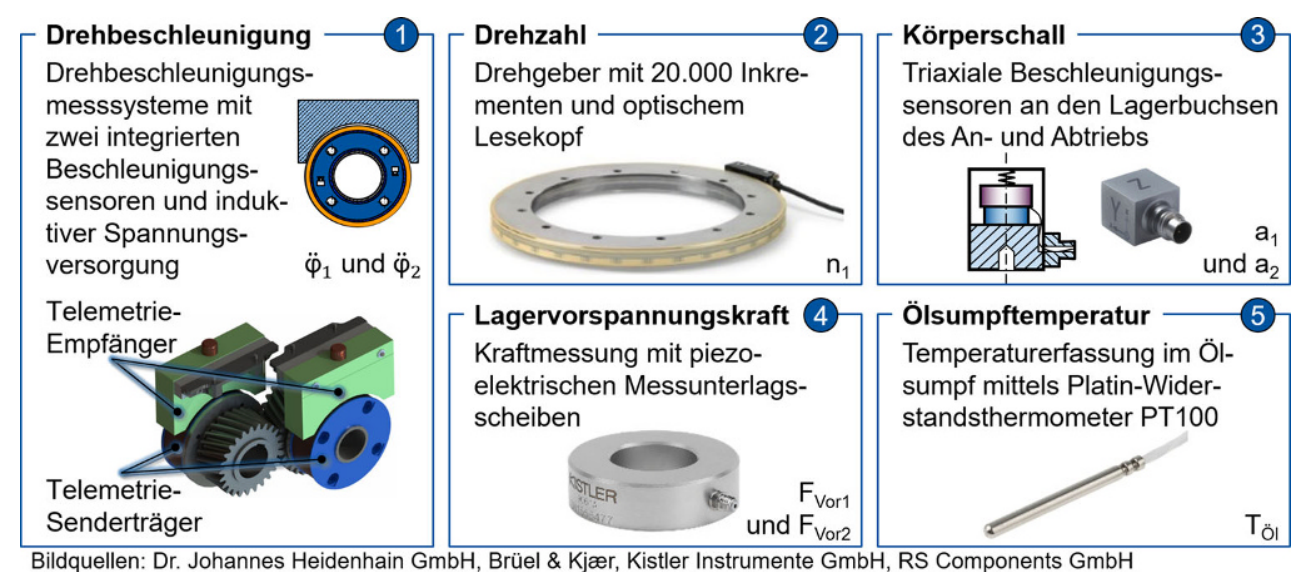

kraft wird in jeder Versuchsreihe das Rasselgeräuschverhalten für die drei mittleren Drehzahlen $\mathrm{n}_{1}=1200 \mathrm{~min}^{-1}$, $\mathrm{n}_{1}=1500 \mathrm{~min}^{-1}$ und $\mathrm{n}_{1}=1800 \mathrm{~min}^{-1}$ untersucht. Zunächst wird die Drehzahl $\mathrm{n}_{1}=1200 \mathrm{~min}^{-1}$ angefahren und der Radsatz wird mittels einer Drehzahlschwankung mit kontinuierlich gesteigerter Schwankungsamplitude $\Delta \mathrm{n}_{1}$ angeregt (vgl. Bereich 1). Die Schwankungsamplitude der Drehzahl wird über eine Dauer von $\Delta \mathrm{t}=20 \mathrm{~s}$ linear auf einen Endwert $\Delta \mathrm{n}_{1}$ gesteigert. Die Rasselanregung erfolgt je Messung bei einer bestimmten Anregungsfrequenz für die Drehzahlschwankung. Die Anregungsfrequenz der Drehzahlschwankung wird bei aufeinanderfolgenden Messungen durch die Abbildung einer 1,5-fachen (1,5. Ordnung), einer 2-fachen (2. Ordnung), einer 2,5-fachen (2,5. Ordnung) und einer 3-fachen Sinusschwingung (3. Ordnung) pro Umdrehung variiert. Anschließend wird der Radsatz mit einer Drehzahlschwankung konstanter Schwankungsamplitude $\Delta \mathrm{n}_{1}$ angeregt (vgl. Bereich 2). Bei einer Drehzahl von $\mathrm{n}_{1}=1200 \mathrm{~min}^{-1}$ wird eine Zeitspanne von $\mathrm{t}=50 \mathrm{~ms}$ für eine Umdrehung benötigt. Es werden unterschiedliche Beträge der konstanten Schwankungsamplitude der Drehzahl für eine Zeitspanne von $\Delta \mathrm{t}=10 \mathrm{~s}$ untersucht. Die Prozedur wird ebenfalls für die Drehzahlen $\mathrm{n}_{1}=1500 \mathrm{~min}^{-1}$ und $\mathrm{n}_{1}=1800 \mathrm{~min}^{-1}$ durchgeführt. Jede Messung wird dreimal wiederholt. Für die drei Versuchsreihen je Schmierstoffspezifikation werden vergleichbare Ölsumpftemperaturen $\mathrm{T}_{\mathrm{ÖI}} \mathrm{zwischen}$ den einzelnen Messpunkten durch ein initiales Warmlaufen und eine gleichbleibende Prüfdauer erzielt, sodass der Einfluss der temperaturabhängigen Schmierstoffviskosität bei der Untersuchung des Schleppmomenteinflusses auf das resultierende Rasselgeräuschverhalten gering gehalten wird. Die maximale Temperaturabweichung zwischen zwei Messpunkten unterschiedlicher Lagervorspannungskraft beträgt $\Delta \mathrm{T}_{\mathrm{O} \mathrm{I}}=3,2^{\circ} \mathrm{C}$ für die Ölspezifikation SAE $85 \mathrm{~W}-90$.

\section{Fertigung der Prüfverzahnung}

In diesem Kapitel wird zunächst auf die Fertigung der Prüfverzahnung im Profilschleifprozess eingegangen. Daraufhin wird die gefertigte Prüfverzahnung vermessen. Die Charakterisierung der Prüfverzahnung erfolgt anhand der gemessenen Zahnflankenabweichungen.

\subsection{Fertigung der Prüfverzahnung im Profilschleifprozess}

Die Prüfverzahnung wird mittels des diskontinuierlichen Profilschleifens gefertigt. Das diskontinuierliche Profilschleifen ist gemäß DIN 8589-11 ein abbildendes Hartfeinbearbeitungsverfahren mit geometrisch unbestimmter Schneide [26]. Das Zahnflankenaufmaß wird durch die Zustellung einer rotierenden Schleifscheibe in radialer Richtung und den axialen Vorschub der Schleifscheibe oder des Werkstücks abgetragen [27]. Das Schleifscheibenprofil stimmt im Achsschnitt mit dem Profil der Zahnlücke im Stirnschnitt bei Geradverzahnungen überein [27]. Die Mikrogeometrie wird im Closed Loop-Verfahren in einer Höfler Stirnrad-Schleifmaschine Viper $500 \mathrm{KW}$ geschliffen, wobei der Schleifmaschine die Soll-Mikrogeometrie vorgegeben wird. Die Zahnlücken des Stirnrades werden im Closed Loop solange wiederholt geschliffen und vermessen, bis die Mikrogeometrie innerhalb der geforderten Toleranzen der Verzahnungsqualität IT 6 liegt.

\subsection{Charakterisierung der gefertigten Prüfverzahnung}

Das Rad und das Ritzel werden auf einem Präzisionsmesszentrum P16 der Firma Klingelnberg vermessen. Die Bewertung der Verzahnungsqualität erfolgt anhand der Vermessung der Profil- und Flankenlinien sowie der TeilungsEinzelabweichung und der Rundlaufabweichung von jeweils vier Zähnen pro Rad gemäß DIN ISO 1328-1 [28]. 
Abb. 8 Arten der Rasselanregung und Messzyklus
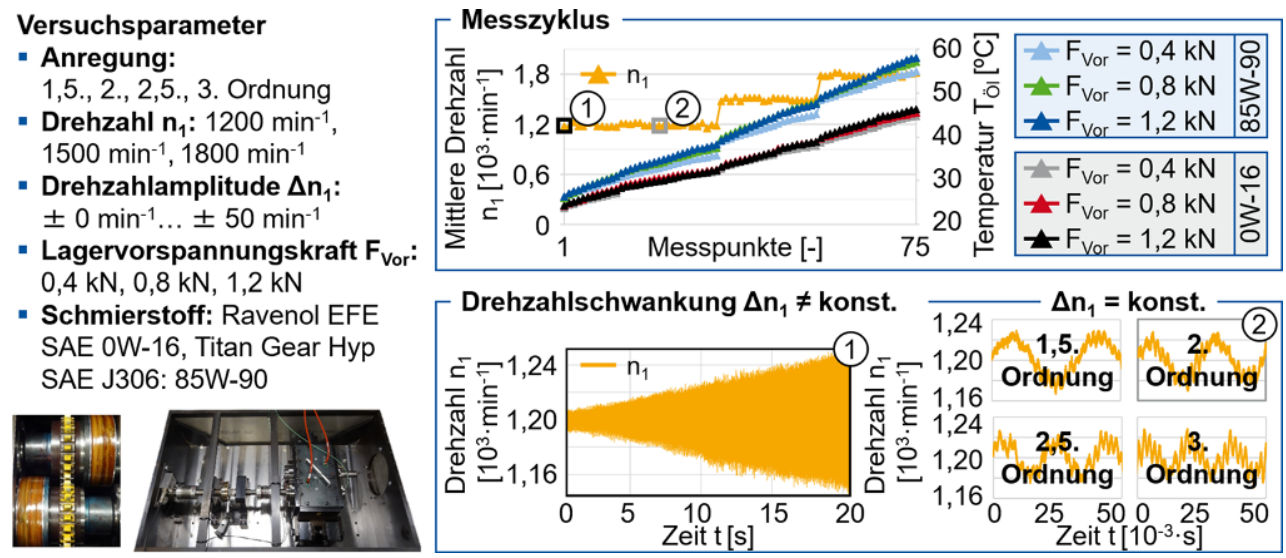

Für die Charakterisierung der Prüfverzahnung werden die arithmetischen Mittel der Profil-Winkelabweichung $\mathrm{f}_{\mathrm{H} \alpha}$, der Profil-Gesamtabweichung $\mathrm{F}_{\alpha}$, der Profil-Formabweichung $\mathrm{f}_{\mathrm{f} \alpha}$, der Profilballigkeit $\mathrm{C}_{\alpha}$, der Teilungsgesamtabweichung $F_{p}$ und der Rundlaufabweichung $F_{r}$ über vier Zähne der linken und der rechten Flanke von Rad und Ritzel sowie die zugehörige Verzahnungsqualität ermittelt. Die Profilund Flankenlinienabweichungen befinden sich bis auf zwei Werte $\mathrm{F}_{\beta}$ der linken Flanke und $\mathrm{f}_{\mathrm{H} \alpha}$ der rechten Flanke des Rades innerhalb der Verzahnungsqualität IT 5. Insgesamt weist das Ritzel die Verzahnungsqualität IT 5 und das Rad die Verzahnungsqualität IT 6 bezüglich der Zahnflankentoleranzen auf. Das diametrale Zweikugelmaß des Ritzels beträgt $M_{\mathrm{dK}, \mathrm{Rit}}=119,767 \mathrm{~mm}$ und das diametrale Zweikugelmaß des Rades beträgt $M_{d K, R a d}=129,690 \mathrm{~mm}$.

\section{Untersuchung des Rasselgeräuschverhaltens von Stirnrädern}

Die Anregungsfrequenz, das Schleppmoment und der Schmierstoff werden bezüglich ihres Einflusses auf das Rasselgeräuschverhalten untersucht. Das Rasselgeräuschverhalten der Prüfverzahnung wird durch die Messung des Körperschalls und der Drehbeschleunigung ausgewertet. Am Ende des Kapitels werden die Versuchsergebnisse zusammengefasst.

\subsection{Einfluss der Anregungsfrequenz auf das Rasselgeräuschverhalten}

Die Anregungsfrequenz der auf die Antriebswelle aufgeprägten Drehzahlschwankung bestimmt die Anzahl an sinusförmigen Schwingungen, die pro Umdrehung vom elektrischen Antriebsmotor vorgegeben wird. Die Abb. 9 zeigt den Körperschallpegel $\mathrm{L}_{\mathrm{a} 2}$ mit dem Bezugswert $\mathrm{a}_{0}=10^{-5} \mathrm{~m} / \mathrm{s}^{2}$ und den Körperschall $\mathrm{a}_{2}$ in $\mathrm{x}$-Richtung am
Abtrieb der Lagerbuchse beispielhaft für eine mittlere Drehzahl von $\mathrm{n}_{1}=1200 \mathrm{~min}^{-1}$ und eine Lagervorspannungskraft $\mathrm{F}_{\mathrm{Vor}}=0,4 \mathrm{kN}$. Der Körperschallpegel $\mathrm{L}_{\mathrm{a} 2}$ ist in der oberen Bildhälfte bei einer Drehzahlschwankung mit linear ansteigender Schwankungsamplitude von $\Delta \mathrm{n}_{1}=0 \mathrm{~min}^{-1}$ auf $\Delta \mathrm{n}_{1}=50 \mathrm{~min}^{-1}$ über eine Dauer von $\Delta \mathrm{t}=20 \mathrm{~s}$ dargestellt und wird als Rasselkurve bezeichnet. Die Drehzahlschwankung wird mit einer Anregung 1,5. Ordnung, 2. Ordnung, 2,5. Ordnung und 3. Ordnung für die Schmierstoffspezifikationen SAE 0W-16 und SAE 85-W90 vorgegeben. $\mathrm{Ab}$ einer bestimmten Schwankungsamplitude der Drehzahl heben die Zahnflanken voneinander ab; dieser Zeitpunkt stellt die Rasselgrenze in der Rasselkurve dar und ist sowohl durch einen starken Anstieg des Körperschallpegels als auch durch eine impulsartige Anregung im Zeitbereich zu erkennen. Die Drehzahlschwankungsamplituden der Rasselgrenze sind basierend auf den Pegelverläufen für das Öl SAE 0W-16 mit niedrigerer Schmierstoffviskosität geringer als für das Öl SAE 85W-90 mit höherer Schmierstoffviskosität. Für den Schmierstoff SAE 0W-16 beginnt die Rasselgrenze bei einer Schwankungsamplitude von $\Delta \mathrm{n}_{1, \text { grenz }}=9 \mathrm{~min}^{-1}$ bei einer Anregung 1,5. Ordnung. Die Rasselgrenzen liegen bei einer Anregung 2. Ordnung, 2,5. Ordnung und 3. Ordnung allesamt bei einer Schwankungsamplitude von $\Delta \mathrm{n}_{1, \text { grenz }}=7,5 \mathrm{~min}^{-1}$ für den Schmierstoff SAE 0W-16. Beim Schmierstoff SAE 85W-90 beträgt die Schwankungsamplitude der Rasselgrenze $\Delta \mathrm{n}_{1, \text { grenz }}=19 \mathrm{~min}^{-1}$ bei einer Anregung 1,5. Ordnung, $\Delta \mathrm{n}_{1, \text { grenz }}=15,5 \mathrm{~min}^{-1}$ bei einer Anregung 2. Ordnung, $\Delta \mathrm{n}_{1, \text { grenz }}=12 \mathrm{~min}^{-1}$ bei einer Anregung 2,5. Ordnung und $\Delta \mathrm{n}_{1, \mathrm{grenz}}=11 \mathrm{~min}^{-1}$ bei einer Anregung 3. Ordnung. Der höherviskose Schmierstoff führt aufgrund eines höheren Schleppmoments erst bei höheren Schwankungsamplituden der Drehzahl zu einem Zahnflankenabheben und weist zudem eine höhere Dämpfung auf. Die Versuchsergebnisse bestätigen die qualitativen Aussagen in der Literatur, nach denen das Schleppmoment zur Verminderung von Rasselgeräuschen vergrößert werden soll und aufgrund der 
Abb. 9 Einfluss der Anregungsfrequenz auf den Körperschall

Abb. 10 Einfluss des Schleppmoments auf den Körperschall und die Drehbeschleunigung
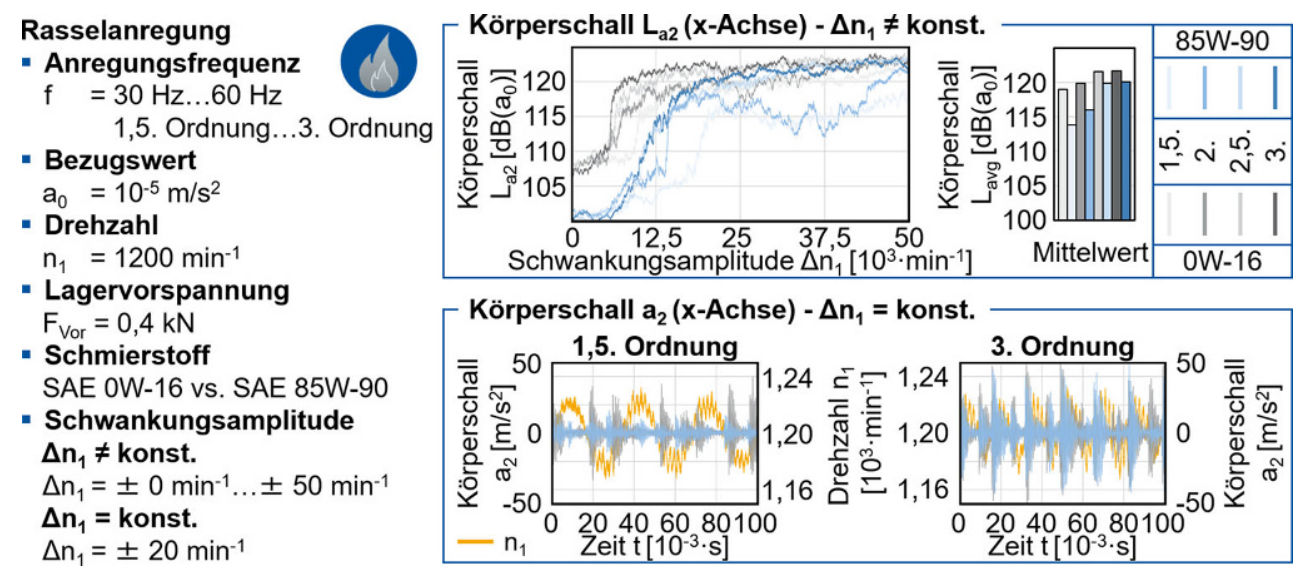
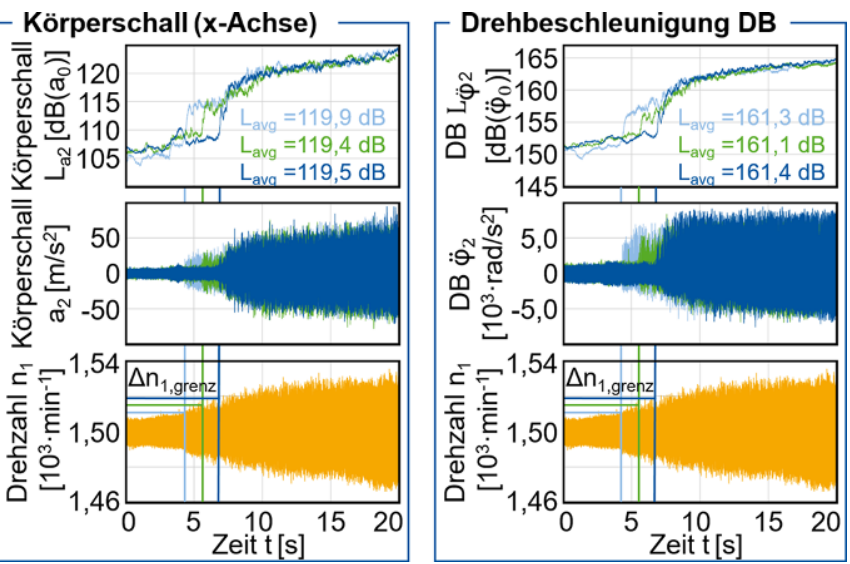

Wirkrichtung ein frühzeitiges Zahnflankenabheben verhindert $[5,20]$. Ab einer Drehzahlschwankungsamplitude von $\Delta \mathrm{n}_{1, \text { grenz }}=25 \mathrm{~min}^{-1}$ bei einer Anregung 2. Ordnung und ab $\Delta \mathrm{n}_{1, \mathrm{grenz}}=30 \mathrm{~min}^{-1}$ bei einer Anregung 1,5. Ordnung sind hingegen kaum noch Dämpfungseffekte des höherviskosen Öls zu erkennen.

In der unteren Bildhälfte sind exemplarisch die zeitlichen Körperschallverläufe $a_{2}$ für zwei Ritzelumdrehungen - dies entspricht einer Zeitspanne von $t=100 \mathrm{~ms}$ bei einer mittleren Drehzahl von $\mathrm{n}_{1}=1200 \mathrm{~min}^{-1}$ - bei einer Drehzahlschwankung mit einer Anregungsfrequenz von $\mathrm{f}=30 \mathrm{~Hz}(1,5$. Ordnung) und mit einer Anregungsfrequenz von $\mathrm{f}=60 \mathrm{~Hz}$ (3. Ordnung) für beide Schmierstoffe linear aufgetragen (Abb. 9). Neben einem deutlichen Anstieg der Körperschallbeträge ist bei zunehmender Anregungsfrequenz zudem eine höhere Anzahl an Stoßimpulsen in der gleichen Zeitspanne zu erkennen. Die Körperschallverläufe zeigen außerdem im Zeitbereich bei Betrachtung der Abklingkurven je Impulsanregung eine höhere Dämpfung bei dem Schmierstoff SAE 85W-90 mit höherer Schmierstoffviskosität. Zusammengefasst kann gesagt werden, dass eine erhöhte Anregungsfrequenz die Schwankungsamplitude der Drehzahl reduziert, bei welcher Rasseln auftritt. Zudem ist eine erhöhte Viskosität für die Rasselgeräuschreduzierung hilfreich.

\subsection{Einfluss des Schleppmoments auf das Rasselgeräuschverhalten}

Das Schleppmoment wird durch die Einstellung von drei unterschiedlichen Lagervorspannungskräften für die angestellte Kegelrollenlagerung variiert und der Einfluss auf den Körperschall und die Drehbeschleunigung am Abtrieb untersucht. Die Abb. 10 zeigt beispielhaft die Pegelverläufe und die linearen Verläufe des Körperschalls und der Drehbeschleunigung für den Schmierstoff SAE $85 \mathrm{~W}-90$ bei einer Drehzahlschwankung mit einer mittleren Drehzahl von $\mathrm{n}_{1}=1500 \mathrm{~min}^{-1}$. Die Schwankungsamplitude wird kontinuierlich von $\Delta \mathrm{n}_{1}=0 \mathrm{~min}^{-1}$ auf $\Delta \mathrm{n}_{1}=30 \mathrm{~min}^{-1}$ über eine Zeitspanne von $\Delta t=20$ s gesteigert.

Das Signal im Zeitbereich und der Gesamtpegel sowohl des Körperschalls als auch der Drehbeschleunigung veranschaulichen, dass die Zahnflanken bei höheren Schleppmomenten erst bei höheren Schwankungsamplituden der Drehzahl voneinander abheben. Die Zeitpunkte für das Erreichen der Rasselgrenze sind sowohl im gepegelten als auch im linearen Zeitverlauf durch einen rapiden Anstieg 
Abb. 11 Einfluss des Schmierstoffs auf den Körperschall
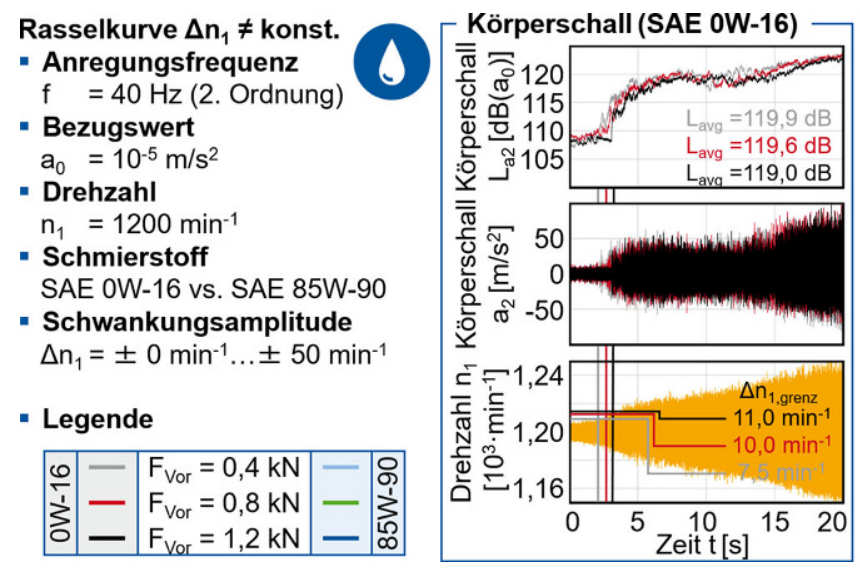

des Körperschalls und der Drehbeschleunigung ersichtlich. Die Rasselgrenze befindet sich für die niedrigste Lagervorspannungskraft von $F_{\text {Vor }}=0,4 \mathrm{kN}$ bei der geringsten Schwankungsamplitude von $\Delta \mathrm{n}_{1, \text { grenz }}=10,5 \mathrm{~min}^{-1}$. Bei der mittleren Lagervorspannungskraft von $\mathrm{F}_{\mathrm{Vor}}=0,8 \mathrm{kN}$ befindet sich die Rasselgrenze bei einer Schwankungsamplitude von $\Delta \mathrm{n}_{1, \text { grenz }}=11,5 \mathrm{~min}^{-1}$ und bei der höchsten Lagervorspannungskraft $\mathrm{F}_{\mathrm{Vor}}=1,2 \mathrm{kN}$ beginnt die Rasselgrenze bei der höchsten Schwankungsamplitude von $\Delta \mathrm{n}_{1, \text { grenz }}=14 \mathrm{~min}^{-1}$. Die Versuchsergebnisse verdeutlichen, dass die Zahnflanken bei einer höheren axialen Lagervorspannungskraft erst ab einer höheren Schwankungsamplitude der Drehzahl $\Delta \mathrm{n}_{1}$ voneinander abheben, da der lastabhängige Reibungsmomentanteil des gesamten Lagerreibungsmoments und folglich das Schleppmoment insgesamt zunehmen, vgl. Gl. 1 und Gl. 2. Zudem sind die Rasselgrenzen des Körperschalls gleich der Rasselgrenzen der Drehbeschleunigung. Insgesamt zeigt die Lagervorspannungskraft keinen signifikanten Einfluss auf das Rasselgeräusch. Lediglich die Rasselgrenze wird durch die Lagervorspannungskraft beeinflusst. Nach dem Überschreiten der Rasselgrenze zeigen sich jedoch keine signifikanten Unterschiede mehr. Die Versuchsergebnisse bestätigen die qualitativen Aussagen in der Literatur, nach denen das Schleppmoment nur in der Nähe der Rasselgrenze einen signifikanten Einfluss auf das Rasselgeräuschverhalten hat [16, 20].

\subsection{Einfluss des Schmierstoffs auf das Rasselgeräuschverhalten}

Der Schmierstoffeinfluss auf das Rasselgeräuschverhalten wird durch die Betrachtung von zwei Schmierstoffspezifikationen unterschiedlicher Viskosität analysiert. Die Abb. 11 zeigt die gepegelten und linearen Verläufe des Körperschalls am Abtrieb bei einer mittleren Drehzahl von $\mathrm{n}_{1}=1200 \mathrm{~min}^{-1}$ und bei verschiedenen Lagervorspannungskräften $\mathrm{F}_{\mathrm{Vor}}$. Die Schwankungsamplitude der Drehzahl wird kontinuierlich von $\Delta \mathrm{n}_{1}=0 \mathrm{~min}^{-1}$ auf $\Delta \mathrm{n}_{1}=50 \mathrm{~min}^{-1}$ über $\Delta \mathrm{t}=20 \mathrm{~s}$ gesteigert.

Die Rasselgrenze beginnt beim niedrigviskoseren Schmierstoff SAE $0 \mathrm{~W}-16$ bei einer eingestellten Lagervorspannungskraft von $\mathrm{F}_{\mathrm{Vor}}=0,4 \mathrm{kN}$ bereits $\mathrm{ab}$ einer Schwankungsamplitude von $\Delta \mathrm{n}_{1, \text { grenz }}=7,5 \mathrm{~min}^{-1}$, während bei gleicher Lagervorspannungskraft die Rasselgrenze beim Schmierstoff $85 \mathrm{~W}-90$ bei einer Schwankungsamplitude $\Delta \mathrm{n}_{1, \text { grenz }}=15,5 \mathrm{~min}^{-1}$ stattfindet. Bei der mittleren Lagervorspannungskraft von $\mathrm{F}_{\mathrm{Vor}}=0,8 \mathrm{kN}$ liegt die Rassegrenze beim Schmierstoff SAE 0W-16 bei einer Schwankungsamplitude von $\Delta \mathrm{n}_{1, \text { grenz }}=10 \mathrm{~min}^{-1}$ und bei der Lagervorspannungskraft $\mathrm{F}_{\mathrm{Vor}}=1,2 \mathrm{kN}$ liegt die Rasselgrenze bei $\Delta \mathrm{n}_{1, \text { grenz }}=11 \mathrm{~min}^{-1}$. Das Zahnflankenabheben beginnt hingegen beim Schmierstoff SAE $85 \mathrm{~W}-90$ erst ab einer Schwankungsamplitude von $\Delta \mathrm{n}_{1, \text { grenz }}=17 \mathrm{~min}^{-1}$ für eine Lagervorspannungskraft von $\mathrm{F}_{\mathrm{Vor}}=0,8 \mathrm{kN}$ und ebenfalls bei $\Delta \mathrm{n}_{1, \text { grenz }}=17 \mathrm{~min}^{-1}$ für eine Lagervorspannungskraft von $\mathrm{F}_{\mathrm{Vor}}=1,2 \mathrm{kN}$. Obwohl eine höhere Ölsumpftemperatur bei den betrachteten Messpunkten mit vergleichbarer Lagervorspannungskraft bei der Schmierstoffspezifikation SAE 85W-90 im Vergleich zum Schmierstoff SAE 0W-16 vorliegt (Abb. 8), ist trotz der Temperaturabhängigkeit ein deutlicher Dämpfungseffekt des höherviskosen Schmierstoffs SAE 85W-90 anhand der niedrigeren Körperschallbeträge und -pegel zu erkennen. Während die quadratisch gemittelten Körperschallpegel beim Schmierstoff SAE 0W-16 zwischen $\mathrm{L}_{\mathrm{avg}}=119,0 \mathrm{~dB}$ und $\mathrm{L}_{\text {avg }}=119,9 \mathrm{~dB}$ liegen, sind die quadratisch gemittelten Körperschallpegel beim Schmierstoff SAE $85 \mathrm{~W}-90$ mindestens um $\Delta \mathrm{L}_{\text {avg }}=1,9 \mathrm{~dB}$ geringer und liegen zwischen $\mathrm{L}_{\mathrm{avg}}=116,0 \mathrm{~dB}$ und $\mathrm{L}_{\mathrm{avg}}=117,1 \mathrm{~dB}$. Die quadratisch gemittelten Körperschallpegel sind beim höherviskosen Schmierstoff SAE 85W-90 geringer aufgrund der viskositätsbedingten höheren Dämpfung beim Aufprall der Zahnflankenoberflächen im Vergleich zum Schmierstoff SAE 0W-16. Der Einfluss der Lagervorspannungskraft auf die quadratisch gemittelten Körperschallpegel ist zwischen den beiden Schmierstoffen vergleichbar: Die 
Abb. 12 Einfluss des Schmierstoffs auf den Körperschall und die Drehbeschleunigung

Abb. 13 Rasselgrenzen und Mittelwerte der Körperschallpegel
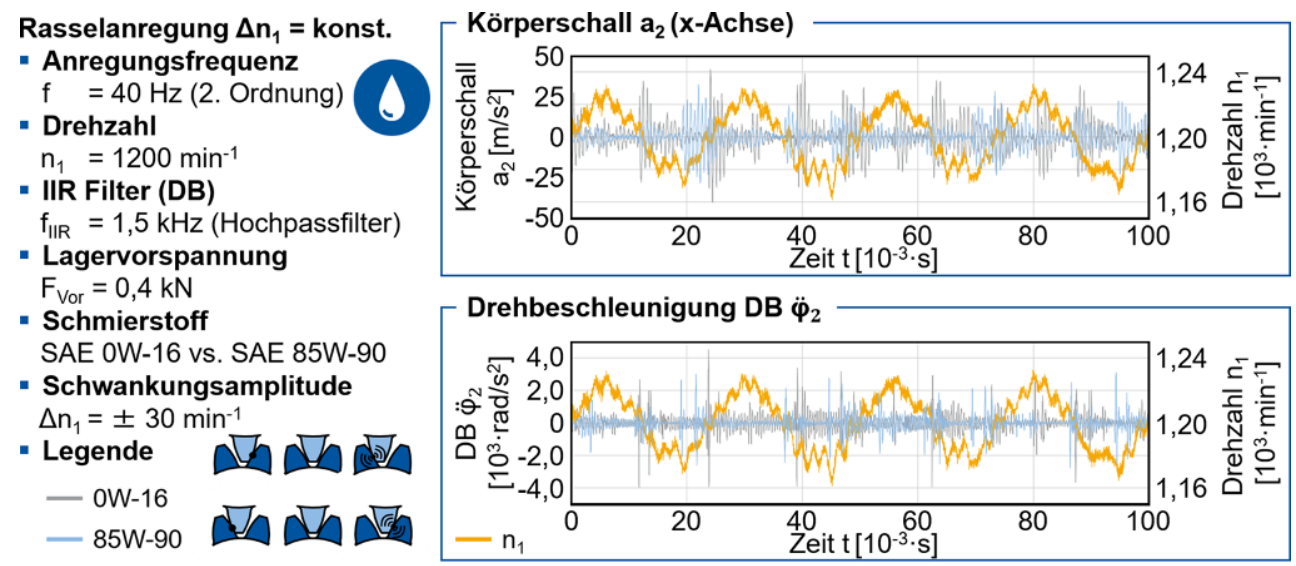
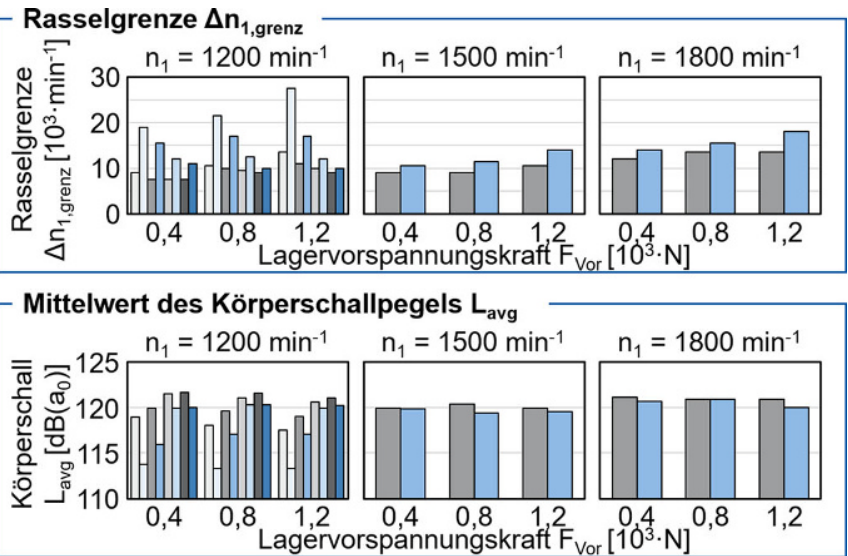

Differenz der quadratisch gemittelten Körperschallpegel beträgt beim Schmierstoff SAE $0 \mathrm{~W}-16 \Delta \mathrm{L}_{\text {avg }}=0,9 \mathrm{~dB}$ und beim Schmierstoff SAE $85 \mathrm{~W}-90 \Delta \mathrm{L}_{\text {avg }}=1,1 \mathrm{~dB}$ zwischen der geringsten Lagervorspannungskraft $F_{\text {Vor }}=0,4 \mathrm{kN}$ und der höchsten Lagervorspannungskraft $\mathrm{F}_{\mathrm{Vor}}=1,2 \mathrm{kN}$. Die Abb. 12 zeigt die Auswirkungen der Impulsanregungen im Zeitbereich auf den Körperschall und die Drehbeschleunigung exemplarisch bei einer Drehzahl von $\mathrm{n}_{1}=1200 \mathrm{~min}^{-1}$ und einer konstanten Schwankungsamplitude der Drehzahl von $\Delta \mathrm{n}_{1}=30 \mathrm{~min}^{-1}$.

Die Stöße treten sowohl im Körperschallverlauf als auch im Drehbeschleunigungsverlauf bevorzugt an den Wendepunkten der sinusförmigen Drehzahlschwankung auf. Dabei wechseln sich Impulse mit niedrigen Körperschallamplituden, bei denen die Zahnflanke des getriebenen Rades auf die Schubflanke des treibenden Rades stößt, mit Impulsen mit höheren Körperschallamplituden ab, bei denen die Zahnflanke des getriebenen Rades mit der Zugflanke des treibenden Rades zusammenstößt, wie nach DoĞAN [5] beschrieben. Die Verdrehflankenstöße weisen beim Vergleich der Schmierstoffe unterschiedlich hoch ausgeprägte Körperschallamplituden auf. Die Stöße im zeitlichen Körperschallverlauf klingen für den Schmierstoff SAE 85W90 schneller ab als beim Schmierstoff SAE 0W-16 und veranschaulichen den höheren Dämpfungseffekt des höherviskosen Schmierstoffs SAE 85W-90.

\subsection{Zusammenfassung der Versuchsergebnisse}

Die Einflüsse der Anregungsfrequenz, des Schleppmoments und des Schmierstoffs auf die Rasselgrenze $\Delta \mathrm{n}_{1, \text { grenz }}$ und die quadratisch gemittelten Körperschallpegel $\mathrm{L}_{\text {avg }}$ sind in Abb. 13 für die Rasselkurven bei den drei untersuchten mittleren Drehzahlen zusammengefasst. Die Schwankungsamplitude wird bei einer mittleren Drehzahl von $\mathrm{n}_{1}=1200 \mathrm{~min}^{-1}$ kontinuierlich von $\Delta \mathrm{n}_{1}=0 \mathrm{~min}^{-1}$ auf $\Delta \mathrm{n}_{1}=50 \mathrm{~min}^{-1}$ gesteigert. Bei den mittleren Drehzahlen $\mathrm{n}_{1}=1500 \mathrm{~min}^{-1}$ und $\mathrm{n}_{1}=1800 \mathrm{~min}^{-1}$ wird die Schwankungsamplitude hingegen von $\Delta \mathrm{n}_{1}=0 \mathrm{~min}^{-1}$ kontinuierlich auf $\Delta \mathrm{n}_{1}=30 \mathrm{~min}^{-1}$ gesteigert.

Die quadratisch gemittelten Körperschallpegel des Schmierstoffs SAE 85W-90 sind bei gleicher Anregungsfrequenz, gleicher mittleren Drehzahl und gleicher Lagervorspannungskraft bis auf einen Messpunkt immer unterhalb der mittleren Körperschallpegel des Schmierstoffs SAE 0W-16. Die größte Differenz der gemittelten Körperschallpegel zwischen den Schmierstoffen liegt mit $\Delta \mathrm{L}_{\text {avg }}=5,13 \mathrm{~dB}$ bei einer mittleren Drehzahl von 
$\mathrm{n}_{1}=1200 \mathrm{~min}^{-1}$ mit einer Anregungsfrequenz $\mathrm{f}=30 \mathrm{~Hz}$ (1,5. Ordnung) und einer Lagervorspannungskraft von $\mathrm{F}_{\mathrm{Vor}}=0,4 \mathrm{kN}$ vor. Bei einer mittleren Drehzahl von $\mathrm{n}_{1}=1800 \mathrm{~min}^{-1}$ mit einer Anregungsfrequenz $\mathrm{f}=60 \mathrm{~Hz}$ (2. Ordnung) und einer Lagervorspannungskraft von $\mathrm{F}_{\mathrm{Vor}}=0,8 \mathrm{kN}$ ist der quadratisch gemittelte Körperschallpegel um $\Delta \mathrm{L}_{\text {avg }}=0,04 \mathrm{~dB}$ beim Schmierstoff SAE 85W-90 leicht höher als beim Schmierstoff SAE 0W-16. Die Schwankungsamplituden der Rasselgrenzen $\Delta \mathrm{n}_{1, \text { grenz }}$ sind bei gleicher Anregungsfrequenz, gleicher mittleren Drehzahl und gleicher Lagervorspannungskraft beim Schmierstoff SAE 85W-90 immer höher als beim Schmierstoff SAE 0W-16. Die Schwankungsamplituden der Rasselgrenzen steigen tendenziell mit abnehmender Anregungsfrequenz und zunehmender Lagervorspannungskraft. Zudem ist eine höhere Abhängigkeit der Anregungsfrequenz auf die Rasselgrenze beim Schmierstoff SAE $85 \mathrm{~W}-90$ bei einer mittleren Drehzahl von $\mathrm{n}_{1}=1200 \mathrm{~min}^{-1}$ im Vergleich zum Schmierstoff SAE 0W-16 zu erkennen: Die Schwankungsamplitude der Rasselgrenze weicht beispielsweise für den Schmierstoff SAE 85W-90 um $\Delta \Delta \mathrm{n}_{1, \text { grenz }}=17,5 \mathrm{~min}^{-1}$ bei einer Lagervorspannungskraft von $F_{\mathrm{Vor}}=1,2 \mathrm{kN}$ beim Vergleich einer Anregung 1,5. Ordnung und 3. Ordnung ab.

Einordnung der Versuchsergebnisse in die Praxis Die experimentelle Analyse der Einflussgrößen auf das Rasselgeräuschverhalten von Stirnrädern zeigt die Herausforderungen im Auslegungsprozess von rasselgeräuschreduzierten Radsätzen sowohl in Steuertrieben als auch in Schaltgetrieben auf. Die Anregungsfrequenz zeigt eine Auswirkung auf das resultierende Rasselgeräuschverhalten von Stirnradgetrieben und wird durch die Art und den Aufbau des Verbrennungsmotors bestimmt. Im Auslegungsprozess muss demnach die Anregungsordnung des Verbrennungsmotors zwingend berücksichtigt werden. Der Trend zum Motordownsizing begünstigt Rasselgeräusche aufgrund erhöhter Drehschwingungen im Antriebsstrang durch steigende Zylinderdrücke und optimierten Verbrauch. Das Downsizing geht neben einer Steigerung der Anregungsamplitude oftmals mit einer Verringerung der Zylinderanzahl einher, die sich beispielsweise bei Viertaktmotoren in Reihenmotorbauform aufgrund der verringerten Anregungsfrequenz günstig auf das Rasselgeräuschverhalten auswirken kann. Die Auswirkungen der Zahnflankenstöße auf das emittierte Geräusch kann durch den Schmierstoff aufgrund von Dämpfungseffekten beeinflusst werden. Der Trend zum dünnflüssigen Motoröl zur Verbrauchsoptimierung stellt aufgrund der erhöhten Rasselneigung eine Herausforderung im Auslegungsprozess von rasselgeräuschreduzierten Motorsteuerrädern dar. Die Versuchsergebnisse zeigen eine Verschiebung der Rasselgrenze zu höheren Drehzahlschwankungsamplituden bei einer Erhöhung des Schleppmoments. Eine
Erhöhung des Schleppmoments geht jedoch aufgrund der zusätzlichen Verlustleistung auch mit einer Verringerung des Wirkungsgrads einher.

\section{Zusammenfassung und Ausblick}

Das Geräuschphänomen des Getrieberasselns tritt infolge von Drehungleichförmigkeiten des Verbrennungsmotors auf, welche zu einem Zahnflankenabheben und impulsartigen Stößen innerhalb des Verdrehflankenspiels der Verzahnung führen. Neben Handschalt- und Doppelkupplungsgetrieben ist eine rasselnde Bewegung zudem bei Steuerrädern im Steuertrieb vorzufinden. In diesem Bericht werden die Einflussgrößen Anregungsfrequenz, Schleppmoment und Schmierstoff auf das Rasselgeräuschverhalten experimentell anhand einer geradverzahnten Stirnradverzahnung untersucht. Das Rasselgeräuschverhalten der Prüfverzahnung wird durch die Erfassung der Drehbeschleunigung, der Drehzahl, des Körperschalls, der Lagervorspannungskraft und der Ölsumpftemperatur untersucht. Der temperaturbedingte Viskositätseinfluss auf das Schleppmoment wird durch eine vergleichbare Ölsumpftemperatur zwischen den Messpunkten gleicher Lagervorspannungskraft gering gehalten. Die Prüfverzahnung wird durch aufgeprägte Drehzahlschwankungen verschiedener Anregungsfrequenzen zur Abbildung der Drehungleichförmigkeit von Viertaktmotoren unterschiedlicher Zylinderanzahl zum Rasseln angeregt. Das Schleppmoment wird durch das Einstellen von drei unterschiedlichen Lagervorspannungskräften verändert und der Prüfablauf wird für zwei Schmierstoffe SAE 0W-16 und SAE 85W-90 durchgeführt. Die Anregungsfrequenz beeinflusst die Anzahl an Verdrehflankenstößen pro Umdrehung und bewirkt tendenziell bei einer Zunahme eine Verringerung der Drehzahlschwankungsamplitude der Rasselgrenze. Der Einfluss der Anregungsfrequenz auf die Rasselgrenze ist beim höherviskosen Schmierstoff SAE 85W-90 höher als beim Schmierstoff SAE 0W-16. Der Dämpfungseffekt des Öls nimmt hingegen mit zunehmender Anregungsfrequenz bei konstanter Schwankungsamplitude der Drehzahl infolge der höheren Winkelbeschleunigungsamplitude ab. Bei steigendem Schleppmoment nimmt die Schwankungsamplitude der Drehzahl tendenziell zu, bei welcher die Prüfverzahnung anfängt zu rasseln. Die Rasselgrenze ist im Pegelverlauf sowohl des Körperschalls als auch der Drehbeschleunigung sowie durch Impulse im Zeitverlauf von Körperschall und Drehbeschleunigung zu erkennen. Der Schmierstoff beeinflusst neben der Schwankungsamplitude der Rasselgrenze auch den Körperschallpegel aufgrund dämpfender Effekte beim impulsartigen Aufeinandertreffen der Zahnflankenoberflächen.

In Folgearbeiten können weitere Einflussgrößen auf das Rasselgeräuschverhalten untersucht werden. Der Einfluss 
des Verdrehflankenspiels kann durch die Variation des Achsabstands, die Fertigung von Prüfverzahnungen mit unterschiedlichen Zahnweiten oder die Verwendung unterschiedlicher Beschichtungssysteme untersucht werden. Zusätzlich kann der Einfluss der Materialdämpfung mittels Prüfverzahnungen mit verschiedenen Radkörpergeometrien oder bestehend aus unterschiedlichen Materialien - beispielsweise Kunststoffe - untersucht werden. Mit simulativen Untersuchungen zur Öldämpfung kann die Rasselanregung in einem MKS-Modell abgebildet werden. Die Rasselgrenze kann hierbei durch die Simulation der Drehbeschleunigung vorhergesagt werden, da sich die Rasselgrenze neben Stoßimpulsen im zeitlichen Körperschallverlauf auch im zeitlichen Verlauf der Drehbeschleunigung wiederfindet. Anhand einer zu entwickelnden Methode kann zudem die Makrogeometrie einer Prüfverzahnung bezüglich des Rasselgeräuschverhaltens ausgelegt werden.

Danksagung Die Autoren danken dem WZL Getriebekreis für die Bereitstellung der finanziellen Mittel zur Durchführung des den vorgestellten Ergebnissen zugrunde liegenden Forschungsprojekts.

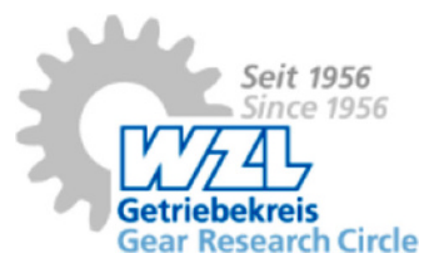

Funding Open Access funding enabled and organized by Projekt DEAL.

Conflict of Interests/Competing interests On behalf of all authors, the corresponding author states that there is no conflict of interest.

Open Access Dieser Artikel wird unter der Creative Commons Namensnennung 4.0 International Lizenz veröffentlicht, welche die Nutzung, Vervielfältigung, Bearbeitung, Verbreitung und Wiedergabe in jeglichem Medium und Format erlaubt, sofern Sie den/die ursprünglichen Autor(en) und die Quelle ordnungsgemäß nennen, einen Link zur Creative Commons Lizenz beifügen und angeben, ob Änderungen vorgenommen wurden.

Die in diesem Artikel enthaltenen Bilder und sonstiges Drittmaterial unterliegen ebenfalls der genannten Creative Commons Lizenz, sofern sich aus der Abbildungslegende nichts anderes ergibt. Sofern das betreffende Material nicht unter der genannten Creative Commons Lizenz steht und die betreffende Handlung nicht nach gesetzlichen Vorschriften erlaubt ist, ist für die oben aufgeführten Weiterverwendungen des Materials die Einwilligung des jeweiligen Rechteinhabers einzuholen.

Weitere Details zur Lizenz entnehmen Sie bitte der Lizenzinformation auf http://creativecommons.org/licenses/by/4.0/deed.de.

\section{Literatur}

1. Zeller P (2018) Handbuch Fahrzeugakustik. Grundlagen, Auslegung, Berechnung, Versuch. Springer Vieweg, Wiesbaden

2. Genuit K (2010) Sound-Engineering im Automobilbereich. Methoden zur Messung und Auswertung von Geräuschen und Schwingungen. Springer, Berlin, Heidelberg, New York
3. Stockmeier M (2008) Entwicklung von klapper- und rasselgeräuschfreien Fahrzeuggetrieben. Diss., Universität Stuttgart

4. Fietkau P (2013) Transiente Kontaktberechnung bei Fahrzeuggetrieben. Diss., Universität Stuttgart

5. Doğan SN (2001) Zur Minimierung der Losteilgeräusche von Fahrzeuggetrieben. Diss., Universität Stuttgart

6. Dion J-L, Le Moyne S, Chevallier G, Sebbah H (2009) Gear impacts and idle gear noise: experimental study and non-linear dynamic model. Mech Syst Signal Process 23(8):2608-2628

7. Genuit K (2001) Störgeräusch. Beurteilung und Katalogisierung von Störgeräuschen bei Verbrennungsmotoren. Abschlussbericht zum Forschungsvorhaben Nr. 720, Heft 715, Forschungsvereinigung Verbrennungskraftmaschinen e.V., Frankfurt a.M.

8. Lang C-H (1997) Losteilgeräusche von Fahrzeuggetrieben. Berichte aus dem Institut für Maschinenelemente, Antriebstechnik, CAD, Dichtungen, Zuverlässigkeit, Bd. 73. Institut für Maschinenelemente, Stuttgart

9. Baumann A (2013) Rasselgeräuschminimierung von Fahrzeuggetrieben durch Getriebeöle. Berichte aus dem Institut für Maschinenelemente, Antriebstechnik, CAD, Dichtungen, Zuverlässigkeit, Bd. 150. IMA, Stuttgart

10. Jörg B (Hrsg) (1988) Abbau von Resonanzschwingungen im Antriebsstrang durch Drehschwingungstilger. VDI-Berichte. VDI, Düsseldorf, S 159-172

11. Ziegler P, Eberhard P (2008) Simulative and experimental investigation of impacts on gear wheels. Comput Methods Appl Mech Eng 197(51-52):4653-4662

12. Tschöke H, Mollenhauer K, Maier R (2018) Handbuch Dieselmotoren. Springer Reference Technik. Springer Vieweg, Wiesbaden

13. Weck M, Fritsch P (1987) Zahnflanken-Hämmern. Ursachen und Auswirkungen des Drehmomentwechsels bei schwingender Belastung von Zahnflanken. Abschlussbericht zum Forschungsvorhaben Nr. 112, Heft 258, Forschungsvereinigung Antriebstechnik e.V., Frankfurt a.M.

14. Wilhelm M, Laurin S, Schmillen K, Spessert B (1990) Structure vibration excitation by timing gear impacts. SAE Tech Pap 99:1-13

15. Tatar A, Karaca C, Subasi O, Tabak MS, Sanliturk KY (2018) Effect of gear loading on idle gear noise of heavy-duty diesel engines. In: International gear conference 2018

16. Rach J (1998) Beitrag zur Minimierung von Klapper- und Rasselgeräuschen von Fahrzeuggetrieben. Diss., Universität Stuttgart

17. Novak W (2010) Geräusch- und Wirkungsgradoptimierung bei Fahrzeuggetrieben durch Festradentkopplung. Diss., Universität Stuttgart

18. Ryborz J (2003) Klapper- und Rasselgeräuschverhalten von Pkwund Nkw-Getrieben. Diss., Universität Stuttgart

19. Hartono EA, Golubev M, Chernoray V (2013) PIV study of fluid flow inside a gearbox, Chalmers University of Technology. In: 10th International Symposium on Particle Image Velocimetry. Delft

20. Weidner G (1991) Klappern und Rasseln von Fahrzeuggetrieben. Diss., Universität Stuttgart

21. Brändlein J (1995) Die Wälzlagerpraxis. Handbuch für die Berechnung und Gestaltung von Lagerungen. Vereinigte Fachverlage, Mainz

22. Klingelnberg AG Höfler Stirnrad-Schleifmaschine Viper $500 \mathrm{KW}$. https://www.klingelnberg.com/geschaeftsbereiche/hoefler/stirnradschleifmaschinen/detailseite/product/viper-500/. Zugegriffen: 17. Nov. 2020

23. Klingelnberg AG Präzisionsmesszentrum P16. https://klingelnberg. com/geschaeftsbereiche/praezisionsmesszentren/praezisions messzentren/detailseite/product/p-16/. Zugegriffen: 17. Nov. 2020

24. Carl CF (2014) Gehörbezogene Analyse und Synthese der vibroakustischen Geräuschanregung von Verzahnungen. Diss., RWTH Aachen University

25. Hesse J (2012) Verzahnungsanregung im Antriebsstrang. Diss., RWTH Aachen University 
26. DIN 8589-11:2003-09. Fertigungsverfahren Spanen - Teil 11: Schleifen mit rotierendem Werkzeug. Einordnung, Unterteilung, Begriffe

27. Brecher C, Klocke F (2017) Zahnrad- und Getriebetechnik. Auslegung - Herstellung - Untersuchung - Simulation. Hanser, München 28. DIN ISO 1328-1:2018-03. Zylinderräder - ISO-Toleranzsystem - Teil 1: Definitionen und zulässige Werte für Abweichungen an Zahnflanken 